\title{
Subharmonic Resonance of Van Der Pol Oscillator with Fractional-Order Derivative
}

\author{
Yongjun Shen, ${ }^{1}$ Peng Wei, ${ }^{1}$ Chuanyi Sui, ${ }^{2}$ and Shaopu Yang ${ }^{1}$ \\ ${ }^{1}$ Department of Mechanical Engineering, Shijiazhuang Tiedao University, No. 17 Bei Erhuan Dong Road, Shijiazhuang, \\ Hebei 050043, China \\ ${ }^{2}$ Key Laboratory of Transportation Tunnel Engineering, Ministry of Education, Southwest Jiaotong University, \\ No. 111 North 2nd Ring Street, Chengdu 610031, China
}

Correspondence should be addressed to Yongjun Shen; shenyongjun@126.com

Received 4 November 2013; Revised 16 January 2014; Accepted 17 January 2014; Published 25 February 2014

Academic Editor: Wang Xing-yuan

Copyright (C) 2014 Yongjun Shen et al. This is an open access article distributed under the Creative Commons Attribution License, which permits unrestricted use, distribution, and reproduction in any medium, provided the original work is properly cited.

\begin{abstract}
The subharmonic resonance of van der Pol (VDP) oscillator with fractional-order derivative is studied by the averaging method. At first, the first-order approximate solutions are obtained by the averaging method. Then the definitions of equivalent linear damping coefficient (ELDC) and equivalent linear stiffness coefficient (ELSC) for subharmonic resonance are established, and the effects of the fractional-order parameters on the ELDC, the ELSC, and the dynamical characteristics of system are also analysed. Moreover, the amplitude-frequency equation and phase-frequency equation of steady-state solution for subharmonic resonance are established. The corresponding stability condition is presented based on Lyapunov theory, and the existence condition for subharmonic resonance (ECSR) is also obtained. At last, the comparisons of the fractional-order and the traditional integer-order VDP oscillator are fulfilled by the numerical simulation. The effects of the parameters in fractional-order derivative on the steadystate amplitude, the amplitude-frequency curves, and the system stability are also studied.
\end{abstract}

\section{Introduction}

Fractional-order calculus has almost the same long history as the traditional integer-order calculus, and it was presented more than 300 years ago. Fractional-order system has a great influence on the things in nature which could be seen, touched, and controlled. Fractional-order calculus developed slowly because it had no obviously practical application for a long time due to the relatively low calculation level in early time. In recent years, fractional-order calculus was paid more and more attention from researchers in different fields and became an international hot topic. Fractionalorder calculus have been studied extensively in the fields of electrochemistry [1], viscoelastic theory [2], automatic control theory [3], signal engineering [4], fluid mechanic [5], quantum mechanics [6], dynamics [7], material science [8], and so forth. At present, there were a lot of scholars studying on the fractional-order calculus and presented important results. For example, Shen et al. [9-11] investigated some linear and nonlinear fractional-order oscillators by averaging method, established the definitions of equivalent linear damping coefficient and equivalent linear stiffness coefficient with fractional-order derivative parameters, and analysed the effects of the fractional-order derivative parameters on the dynamical characteristics of system. Gorenflo and Abdel-Rehim [12], Jumarie [13], Ishteva et al. [14], and Malinowska and Torres [15], respectively studied the definitions and numerical methods of fractional-order calculus for Grünwald-Letnikov, Riemann-Liouville, and Caputo. Xu et al. [16] combined Lindstedt-Poincare method and multiscale method to study fractional-order Duffing oscillator to harmonic excitation with random phase and analyzed the stochastic jump and bifurcation in the oscillator. Li et al. [1719] have done a lot of researches in the mathematical theory of fractional-order calculus and established some efficient numerical algorithms. By using the idea of stability switch, Wang et al. [20, 21] investigated a linear single degree-offreedom (SDOF) oscillator with fractional-order derivative and obtained some important conclusions about the equilibriums and the composition of the solution. Atanackovic and 
Stankovic [22], J.-H. Chen and W.-C. Chen [23], Lu [24], and Cao et al. [25] numerically studied different fraction-order nonlinear oscillators, and some complicated phenomena in those oscillators were found, such as bifurcation, chaos, hyperchaos, and fractal. Huang and Jin [26] and Chen and Zhu [27] studied different fractional-order oscillators with random excitation by different methods and obtained the important statistical properties of those oscillators. Wang et al. [28-34] studied different fractional-order nonlinear oscillators by different methods, and obtained some important conclusions.

Van der Pol established the famous van der Pol (VDP) equation [35] to describe the tube oscillator in LC circuit since 1928. After that, the VDP oscillator became one of the basic dynamical equations in mathematical and physical field and was important in the self-excited oscillation theory. Although its form was relatively simple, the VDP oscillator received extensive attentions due to its special dynamical characteristics of the nonlinear damping.

Comparing with the traditional integer-order system, the fractional-order system has more advantages and is much closer to the real nature of the world. The VDP oscillator with fractional-order derivative can be more accurate to reflect the real properties of many nonlinear oscillators. Some classical analytical methods for nonlinear system, including their improved version, such as harmonic balance method [36], multiscale method [37, 38], perturbation method [39], averaging method $[9,40,41]$, and KBM method [42] may be applied into the fractional-order oscillator.

In this paper, the subharmonic resonance of fractionalorder VDP oscillator is analytically researched by averaging method. In Section 2, the approximate solution for subharmonic resonance of the fractional-order VDP oscillator is investigated, where the effects of the fractional-order derivative on the system damping and stiffness are formulated as equivalent linear damping coefficient (ELDC) and equivalent linear stiffness coefficient (ELSC). Section 3 presents the stability condition for steady-state solution based on Lyapunov theory and the existence condition in subharmonic resonance (ECSR). The effects of the fractional-order parameters on the stability condition for steady-state solution and the ECSR are also analyzed. In Section 4, the effects of the fractionalorder parameters on the ECSR, the steady-state amplitude, the amplitude-frequency curves, and the system stability are studied by the numerical method. The comparisons between the integer-order with the fractional-order system are also fulfilled in this section. Finally, the main conclusions of this paper are drawn in Section 5.

\section{The Approximately Analytical Solution for Subharmonic Resonance of Fractional-Order VDP Oscillator}

The mathematical model of VDP oscillator with fractionalorder derivative is established as

$$
\begin{gathered}
m \ddot{x}(t)+k x(t)+\alpha_{1}\left[x^{2}(t)-1\right] \dot{x}(t) \\
+K_{1} D^{p}[x(t)]=F_{1} \cos (\omega t)
\end{gathered}
$$

where $m, k, \alpha_{1}, F_{1}, \omega$ are the system mass, linear stiffness coefficient, nonlinear damping coefficient, excitation amplitude, and excitation frequency, respectively. $K_{1} D^{p}[x(t)]$ is the $p$ order derivative of $x(t)$ to $t$ with the fractional coefficient $K_{1}\left(K_{1}>0\right)$ and the fractional order $p(0 \leq p \leq 1)$. There are several definitions for fractional-order derivative, and Caputo's definition [30] is adopted here

$$
D^{p}[z(t)]=\frac{1}{\Gamma(1-p)} \int_{0}^{t} \frac{z^{\prime}(u)}{(t-u)^{p}} d u
$$

where $\Gamma(z)$ is gamma function satisfying $\Gamma(z+1)=z \Gamma(z)$.

Using the transformation of coordinates as follows:

$$
\omega_{0}=\sqrt{\frac{k}{m}}, \quad \varepsilon \alpha=\frac{\alpha_{1}}{m}, \quad \varepsilon k_{1}=\frac{K_{1}}{m}, \quad F=\frac{F_{1}}{m} .
$$

Equation (1) becomes

$$
\begin{aligned}
\ddot{x}(t) & +\omega_{0}^{2} x(t)+\varepsilon \alpha\left[x^{2}(t)-1\right] \dot{x}(t) \\
& +\varepsilon k_{1} D^{p}[x(t)]=F \cos (\omega t) .
\end{aligned}
$$

In order to study the $1 / 3$ subharmonic resonance of fractional-order VDP oscillator; that is, $\omega \approx 3 \omega_{0}$, one could introduce

$$
\frac{1}{9} \omega^{2}=\omega_{0}^{2}+\varepsilon \sigma
$$

where $\sigma$ is the detuning factor for excitation frequency. Then, (4) becomes

$$
\begin{array}{r}
\ddot{x}(t)+\frac{1}{9} \omega^{2} x(t)=\varepsilon\left\{\sigma x(t)+\alpha\left[1-x^{2}(t)\right] \dot{x}(t)\right. \\
\left.-k_{1} D^{p}[x(t)]\right\}+F \cos (\omega t) .
\end{array}
$$

Suppose that (6) has the solution as

$$
\begin{gathered}
x=a \cos \varphi+B \cos (\omega t), \\
\dot{x}=-\frac{1}{3} a \omega \sin \varphi-B \omega \sin (\omega t),
\end{gathered}
$$

where $\varphi=(1 / 3) \omega t+\theta$ and $B=F /\left(\omega_{0}^{2}-\omega^{2}\right) \approx-\left(9 F / 8 \omega^{2}\right)$. According to the averaging method, we could obtain

$$
\begin{aligned}
& \dot{a}=-\frac{3}{\omega}\left[P_{1}(a, \theta)+P_{2}(a, \theta)\right] \sin \varphi, \\
& a \dot{\theta}=-\frac{3}{\omega}\left[P_{1}(a, \theta)+P_{2}(a, \theta)\right] \cos \varphi,
\end{aligned}
$$

where

$$
\begin{aligned}
P_{1}(a, \theta)= & \varepsilon[\sigma a \cos \varphi+\sigma B \cos (3 \varphi-3 \theta)] \\
& -\varepsilon \alpha\left[\frac{1}{3} a \omega \sin \varphi+B \omega \sin (3 \varphi-3 \theta)\right] \\
& \times\left\{1-[a \cos \varphi+B \cos (3 \varphi-3 \theta)]^{2}\right\}, \\
P_{2}(a, \theta)= & -\varepsilon k_{1} D^{p}[a \cos \varphi+B \cos (3 \varphi-3 \theta)] .
\end{aligned}
$$


We could apply the standard averaging procedure to the right sides of $(8 \mathrm{a})$ and $(8 \mathrm{~b})$ in time interval $[0, T]$, that means

$$
\begin{gathered}
\dot{a}=-\frac{3}{T \omega} \int_{0}^{T}\left[P_{1}(a, \theta)+P_{2}(a, \theta)\right] \sin \varphi d \varphi, \\
\dot{a}=-\frac{3}{T \omega} \int_{0}^{T}\left[P_{1}(a, \theta)+P_{2}(a, \theta)\right] \cos \varphi d \varphi .
\end{gathered}
$$

In the procedure of integrating and averaging, one could select the time terminal $T$ as $T=2 \pi$ if $P_{i}(a, \theta)(i=1,2)$ is periodic function, or $T=\infty$ if $P_{i}(a, \theta)(i=1,2)$ is aperiodic one. Hereby, one could obtain the simplified forms of the first part for (10a) and (10b)

$$
\begin{aligned}
\dot{a}_{1} & =-\frac{3}{2 \pi \omega} \int_{0}^{2 \pi} P_{1}(a, \theta) \sin \varphi d \varphi \\
& =\frac{\varepsilon \alpha a}{2}-\frac{\varepsilon \alpha}{8}\left[a^{3}+a^{2} B \cos (3 \theta)+2 a B^{2}\right] \\
a \dot{\theta}_{1} & =-\frac{3}{2 \pi \omega} \int_{0}^{2 \pi} P_{1}(a, \theta) \cos \varphi d \varphi \\
& =-\frac{3 \varepsilon \sigma a}{2 \omega}+\frac{\varepsilon \alpha a^{2} B}{8} \sin (3 \theta) .
\end{aligned}
$$

In order to calculate the second part for (10a) and (10b)

$$
\begin{aligned}
\dot{a}_{2} & =-\lim _{T \rightarrow \infty} \frac{3}{T \omega} \int_{0}^{T} P_{2}(a, \theta) \sin \varphi d \varphi \\
& =\lim _{T \rightarrow \infty} \frac{3 \varepsilon k_{1}}{T \omega} \int_{0}^{T} D^{p}[a \cos \varphi+B \cos (3 \varphi-3 \theta)] \sin \varphi d \varphi,
\end{aligned}
$$

$$
\begin{aligned}
a \dot{\theta}_{2} & =-\lim _{T \rightarrow \infty} \frac{3}{T \omega} \int_{0}^{T} P_{2}(a, \theta) \cos \varphi d \varphi \\
& =\lim _{T \rightarrow \infty} \frac{3 \varepsilon k_{1}}{T \omega} \int_{0}^{T} D^{p}[a \cos \varphi+B \cos (3 \varphi-3 \theta)] \cos \varphi d \varphi,
\end{aligned}
$$

we introduce two important formulae as follows:

$$
\begin{aligned}
& B_{1}=\lim _{T \rightarrow \infty} \int_{0}^{T} \frac{\sin (\omega t)}{t^{p}} d t, \\
& B_{2}=\lim _{T \rightarrow \infty} \int_{0}^{T} \frac{\cos (\omega t)}{t^{p}} d t .
\end{aligned}
$$

Based on the residue theorem and contour integration, one can obtain

$$
\begin{aligned}
& B_{1}=\frac{\omega^{p-1} \Gamma(2-p)}{1-p} \cos \left(\frac{p \pi}{2}\right)=\omega^{p-1} \Gamma(1-p) \cos \left(\frac{p \pi}{2}\right), \\
& B_{2}=\frac{\omega^{p-1} \Gamma(2-p)}{1-p} \sin \left(\frac{p \pi}{2}\right)=\omega^{p-1} \Gamma(1-p) \sin \left(\frac{p \pi}{2}\right) .
\end{aligned}
$$

After some complicated but standard computation, we could obtain

$$
\begin{aligned}
& \dot{a}_{2}=-\frac{\varepsilon a k_{1}(\omega / 3)^{p-1}}{2} \sin \left(\frac{p \pi}{2}\right), \\
& a \dot{\theta}_{2}=\frac{\varepsilon a k_{1}(\omega / 3)^{p-1}}{2} \cos \left(\frac{p \pi}{2}\right) .
\end{aligned}
$$

Combining (11a) and (11b) with (15a) and (15b), one could establish the simplified standard equation as

$$
\begin{gathered}
\dot{a}=\frac{\varepsilon \alpha a}{2}-\frac{\varepsilon \alpha}{8}\left(a^{3}+a^{2} B \cos 3 \theta+2 a B^{2}\right) \\
-\frac{\varepsilon a k_{1}(\omega / 3)^{p-1}}{2} \sin \left(\frac{p \pi}{2}\right), \\
a \dot{\theta}=-\frac{3 \varepsilon \sigma a}{2 \omega}+\frac{\varepsilon \alpha a^{2} B}{8} \sin 3 \theta+\frac{\varepsilon a k_{1}(\omega / 3)^{p-1}}{2} \cos \left(\frac{p \pi}{2}\right) .
\end{gathered}
$$

If we substitute the parameters with the original ones, (16a) and (16b) become

$$
\begin{gathered}
\dot{a}=-\frac{\alpha_{1} a^{2} B}{8 m} \cos (3 \theta)-\frac{\alpha_{1}\left(a^{3}+2 a B^{2}\right)}{8 m}-\frac{a}{2 m} C(p), \\
a \dot{\theta}=\frac{\alpha_{1} a^{2} B}{8 m} \sin (3 \theta)-\frac{a \omega}{6}+\frac{3 a}{2 m \omega} K(p),
\end{gathered}
$$

where

$$
\begin{gathered}
C(p)=-\alpha_{1}+K_{1}\left(\frac{\omega}{3}\right)^{p-1} \sin \left(\frac{p \pi}{2}\right) \\
K(p)=k+K_{1}\left(\frac{\omega}{3}\right)^{p} \cos \left(\frac{p \pi}{2}\right) .
\end{gathered}
$$

We refer to these two combined new parameters as equivalent linear damping coefficient (ELDC) and equivalent linear stiffness coefficient (ELSC), respectively.

From (18a) and (18b), we can know that fractional-order parameters have important influence on the ELDC and the ELSC. It is easy to see that the equivalent linear damping and stiffness coefficients are all monotonically increasing function of the fractional coefficient $K_{1}$. Therefore, the fractional coefficient $K_{1}$ will affect the system response amplitude by the form of ELDC and affect the system resonance frequency by the form of ELSC. The effects of the fractional order $p$ on the 
ELDC and ELSC are much more complicated. These effects are implemented in the product form of an exponential and a trigonometric function. We could take the partial derivatives of the ELDC and ELSC with respect to $p$, and the results are as follows:

$$
\begin{aligned}
& \frac{\partial C(p)}{\partial p} \\
& =K_{1}\left[\left(\frac{\omega}{3}\right)^{p-1} \ln \left(\frac{\omega}{3}\right) \sin \left(\frac{p \pi}{2}\right)+\frac{\pi}{2}\left(\frac{\omega}{3}\right)^{p-1} \cos \left(\frac{p \pi}{2}\right)\right],
\end{aligned}
$$

$$
\begin{aligned}
& \frac{\partial K(p)}{\partial p} \\
& =K_{1}\left[\left(\frac{\omega}{3}\right)^{p} \ln \left(\frac{\omega}{3}\right) \cos \left(\frac{p \pi}{2}\right)-\frac{\pi}{2}\left(\frac{\omega}{3}\right)^{p} \sin \left(\frac{p \pi}{2}\right)\right] .
\end{aligned}
$$

From (19a) and (19b), we can know that the partial derivatives of the ELDC and ELSC to the fractional order $p$ are related to the excitation frequency besides being related to $p$. When the fractional order $p$ is changed from 0 to 1 , $(\omega / 3)^{p}$ and $(\omega / 3)^{p-1}$ will be decreased along with the increase of $p$ if $\omega / 3<1$. On the contrary, $(\omega / 3)^{p}$ and $(\omega / 3)^{p-1}$ will be increased along with the increase of $p$ if $\omega / 3>1$. Because the natural frequency of the selected system in this paper is $\omega_{0}=3$, we can know that $\omega / 3>1$ based on the relationship between $\omega$ and $\omega_{0}$. From (19a), one can conclude that $\partial C(p) / \partial p$ is always positive when $0<p<1$. That means that the ELDC is a monotonically increasing function of the fractional order $p$, and the fractional order $p$ will affect the system response amplitude by the form of ELDC. We can also obtain the critical value for the fractional order $p_{c}=$ 0.3885 from (19b). Through the analysis, we know that the ELSC will attain the maximum value and the corresponding resonance frequency is the largest at the critical value for the fractional order VDP oscillator. It could also be concluded that the ELSC and the corresponding resonance frequency will be increased along with the increase of $p$ if $0<p<p_{c}$ and will be decreased along with the increase of $p$ if $p_{c}<p<$ 1 .

From the above analysis, we can draw the following conclusions. When $p=0$, the ELDC is the minimum value as $-\alpha_{1}$, and the ELSC is $k+K_{1}$. When the fractional order is changed as the critical value as $p_{c}$, the ELSC will attain the maximum value. When $p=1$, the ELDC is the maximum value as $K_{1}-\alpha_{1}$, and the ELSC is the minimum value as $k$. Accordingly, when $p \rightarrow 0$, we can know that the ELDC is very small, so that the corresponding system response amplitude will be very large. When $p \rightarrow 1$, we can find that the ELDC is very large, and the corresponding resonance frequency is close to the natural frequency of the original integer-order system.

\section{Existence Condition for 1/3 Subharmonic Resonance and Stability Condition for the Approximate Solution}

3.1. Existence Condition for $1 / 3$ Subharmonic Resonance. Now we study the steady-state solution, which is more important and meaningful in vibration engineering. Letting $\dot{a}=0$ and $a \dot{\theta}=0,(17 \mathrm{a})$ and (17b) become

$$
\begin{gathered}
-\alpha_{1}\left(\bar{a}^{2}+2 B^{2}\right)-4 C(p)=\alpha_{1} \bar{a} B \cos (3 \bar{\theta}), \\
4 m \omega^{2}-36 K(p)=3 \alpha_{1} \bar{a} B \omega \sin (3 \bar{\theta}) .
\end{gathered}
$$

Eliminating $\bar{\theta}$ from (20) and (21), one could obtain the amplitude-frequency equation as

$$
\left[\frac{\alpha_{1} \bar{a}^{2}}{4}+\frac{\alpha_{1} B^{2}}{2}+C(p)\right]^{2}+\frac{9}{\omega^{2}}\left[K(p)-\frac{m \omega^{2}}{9}\right]^{2}=\frac{\alpha_{1}^{2} \bar{a}^{2} B^{2}}{16} \text {. }
$$

Defining the amplitude parameter as

$$
\rho \stackrel{\text { def }}{=} \frac{\bar{a}^{2}}{4}
$$

one could obtain another form of the amplitude-frequency equation as

$$
\left[\alpha_{1} \rho+\frac{\alpha_{1} B^{2}}{2}+C(p)\right]^{2}+\frac{9}{\omega^{2}}\left[K(p)-\frac{m \omega^{2}}{9}\right]^{2}=\frac{\alpha_{1}^{2} B^{2} \rho}{4} .
$$

According to (20), (21), and (23), we also obtain the phasefrequency equation as

$$
\bar{\theta}=\frac{1}{3} \arctan \left\{\frac{3\left[K(p)-\left(m \omega^{2} / 9\right)\right]}{\omega\left[\alpha_{1} \rho+\left(\alpha_{1} B^{2} / 2\right)+C(p)\right]}\right\} .
$$

Expanding (24), one could get

$$
\begin{aligned}
\alpha_{1}^{2} \rho^{2}+\{2 & \left.\alpha_{1}\left[\frac{\alpha_{1} B^{2}}{2}+C(p)\right]-\frac{\alpha_{1}^{2} B^{2}}{4}\right\} \rho \\
+ & {\left[\frac{\alpha_{1} B^{2}}{2}+C(p)\right]^{2}+\frac{9}{\omega^{2}}\left[K(p)-\frac{m \omega^{2}}{9}\right]^{2}=0 . }
\end{aligned}
$$

Supposing that

$$
\begin{aligned}
& A_{1}=\alpha_{1}^{2}, \quad A_{2}=2 \alpha_{1}\left[\frac{\alpha_{1} B^{2}}{2}+C(p)\right]-\frac{\alpha_{1}^{2} B^{2}}{4}, \\
& A_{3}=\left[\frac{\alpha_{1} B^{2}}{2}+C(p)\right]^{2}+\frac{9}{\omega^{2}}\left[K(p)-\frac{m \omega^{2}}{9}\right],
\end{aligned}
$$

Equation (26) becomes

$$
A_{1} \rho^{2}+A_{2} \rho+A_{3}=0 .
$$


One could obtain the amplitude parameter as

$$
\rho=\frac{-A_{2} \pm \sqrt{A_{2}^{2}-4 A_{1} A_{3}}}{2 A_{1}} .
$$

We can establish the existence condition for subharmonic resonance (ECSR) based on (29) as follows:

$$
A_{2}<0, \quad A_{2}^{2}-4 A_{1} A_{3} \geq 0 .
$$

Expanding (30), we get another form of the ECSR as

$$
\begin{gathered}
\frac{\alpha_{1}^{2} B^{2}}{4}-2 \alpha_{1}\left[\frac{\alpha_{1} B^{2}}{2}+C(p)\right]>0, \\
\frac{\alpha_{1}^{4} B^{4}}{16}-\alpha_{1}^{3} B^{2}\left[\frac{\alpha_{1} B^{2}}{2}+C(p)\right]-\frac{36 \alpha_{1}^{2}}{\omega^{2}}\left[K(p)-\frac{m \omega^{2}}{9}\right]^{2} \geq 0 .
\end{gathered}
$$

From (31a) and (31b), it is easy to see that the condition in (31b) contains the condition in (31a). We simplify (31b) and obtain the ECSR as

$$
\begin{gathered}
\alpha_{1}^{2} B^{4} \omega^{2}-16 \alpha_{1} B^{2} \omega^{2}\left[\frac{\alpha_{1} B^{2}}{2}+C(p)\right] \\
-576\left[K(p)-\frac{m \omega^{2}}{9}\right]^{2} \geq 0 .
\end{gathered}
$$

Through the analysis of (32), we can get to know that the fractional-order parameters will affect the ECSR by the form of the ELDC and ELSC. The ECSR will be decreased along with the increase of the ELDC and ELSC. Taking into account the effects of the fractional-order parameters on the ELDC and the ELSC, we can draw the conclusions that the larger the fractional-order parameters are, the smaller the ECSR is.

3.2. Stability Analysis for the Steady-State Solution. From (26), we get to know that there may be two steady-state solutions for (26) generally. In order to determine which solutions can be achieved in practice, we should analyze the stability of these steady-state solutions. Letting $a=\bar{a}+\Delta a$ and $\theta=\bar{\theta}+\Delta \theta$ and substituting them into (17a) and (17b), that yields

$$
\begin{aligned}
\frac{d \Delta a}{d t}= & {\left[-\frac{\alpha_{1} \bar{a} B \cos (3 \bar{\theta})}{4 m}-\frac{\alpha_{1}\left(3 \bar{a}^{2}+2 B^{2}\right)}{8 m}-\frac{C(p)}{2 m}\right] \Delta a } \\
& +\frac{3 \alpha_{1} \bar{a}^{2} B \sin (3 \bar{\theta})}{8 m} \Delta \theta \\
\frac{d \Delta \theta}{d t} & =\frac{\alpha_{1} B \sin (3 \bar{\theta})}{8 m} \Delta a+\frac{3 \alpha_{1} \bar{a} B \cos (3 \bar{\theta})}{8 m} \Delta \theta
\end{aligned}
$$

Based on (20) and (21), one could eliminate $\bar{\theta}$ from (33a) and (33b) and obtain the characteristic determinant as

$$
\operatorname{det}\left(\begin{array}{cc}
D_{1}-\lambda & -\frac{9 \bar{a}}{2 m \omega}\left[K(p)-\frac{m \omega^{2}}{9}\right] \\
-\frac{3}{2 \bar{a} m \omega}\left[K(p)-\frac{m \omega^{2}}{9}\right] & D_{2}-\lambda
\end{array}\right)=0 \text {, }
$$

where

$$
\begin{aligned}
& D_{1}=\frac{1}{2 m}\left[C(p)+\frac{\alpha_{1} B^{2}}{2}-\alpha_{1} \rho\right], \\
& D_{2}=-\frac{3}{2 m}\left[C(p)+\frac{\alpha_{1} B^{2}}{2}+\alpha_{1} \rho\right] .
\end{aligned}
$$

Then, one could obtain the characteristic equation as follows:

$$
\lambda^{2}-\left(D_{1}+D_{2}\right) \lambda+D_{1} D_{2}-\frac{27}{4 m^{2} \omega^{2}}\left[K(p)-\frac{m \omega^{2}}{9}\right]^{2}=0 \text {. }
$$

From (36), one could obtain the stability conditions for the steady-state solution based on Lyapunov theory as follows:

$$
D_{1}+D_{2}<0, \quad D_{1} D_{2}-\frac{27}{4 m^{2} \omega^{2}}\left[K(p)-\frac{m \omega^{2}}{9}\right]^{2}>0 \text {. }
$$

Expanding (37), one could obtain the equivalent stability conditions as

$$
\begin{aligned}
N(p) \stackrel{\text { def }}{=} 2 \alpha_{1} \rho+\frac{\alpha_{1} B^{2}}{2}+C(p) \\
\quad=\alpha_{1}\left(2 \rho+\frac{B^{2}}{2}-1\right)+K_{1}\left(\frac{\omega}{3}\right)^{p-1} \sin \left(\frac{p \pi}{2}\right)>0,
\end{aligned}
$$

$$
\begin{aligned}
R(p) \stackrel{\text { def }}{=} & \alpha_{1}^{2} \rho^{2}-\left[N(p)-2 \alpha_{1} \rho\right]^{2} \\
& -\frac{9}{\omega^{2}}\left[K(p)-\frac{m \omega^{2}}{9}\right]^{2}>0,
\end{aligned}
$$

where $N(p)$ and $R(p)$ are, respectively, defined as equivalent nonlinear damping coefficient (ENDC) and nonlinear stability parameter (NSP) for subharmonic resonance.

From the stability conditions for the steady-state solution, one could find that there will be stable solution if and only if $N(p)$ and $R(p)$ are positive at the same time. The other three cases, that is, $N(p)>0$ and $R(p)<0, N(p)<0$ and $R(p)>0$, and $N(p)<0$ and $R(p)<0$ are all corresponding to unstable solution. The ENDC is defined under steady state and corresponds to the nonlinear damping term of the fractional-order VDP oscillator.

From (38a) and (38b), we can know that the ENDC is related to the fractional-order parameters, the steadystate amplitude, the excitation amplitude, and excitation frequency. It is very interesting that the NSP is affected by both the ENDC and the ELSC at the same time, and it also has a relationship with the steady-state amplitude and the excitation frequency. When $N(p)=0$ and $R(p)=0, \rho$ will approach the critical value between stable and unstable case. The ENDC and NSP determine the stability of the steadystate amplitude and are affected by the steady-state amplitude in return. The research on system stability is helpful to improve the relative stability of steady-state solution, and it can make the oscillator withstand greater variation range of system parameters so as to improve the robustness of the dynamical system. 


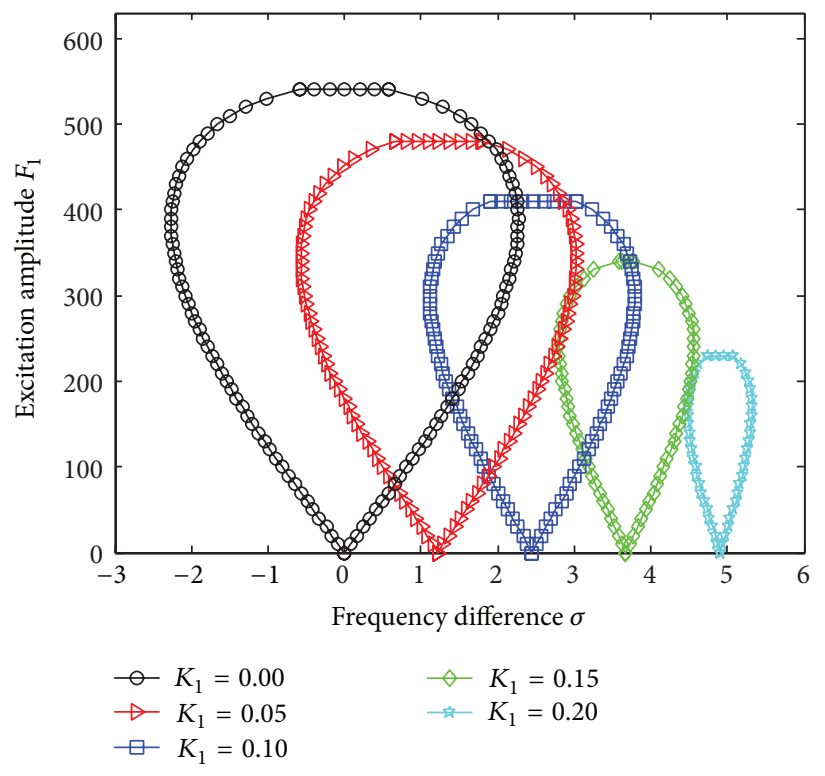

(a)

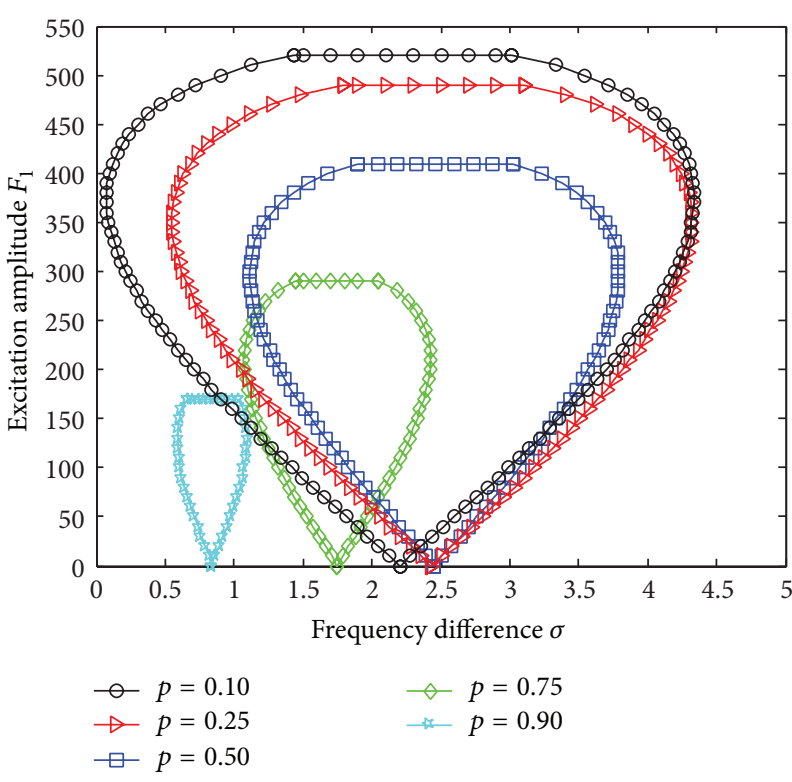

(b)

FIGURE 1: Effects of the fractional-order parameters on the existence condition for subharmonic resonance: (a) $p=0.5$, (b) $K_{1}=0.1$.

\section{Numerical Simulation and Study on the Fractional-Order Parameters}

4.1. Effects of Fractional-Order Parameters on the ECSR. It is important to determine the value ranges of the excitation amplitude and frequency satisfying the ECSR. In order to illustrate the effects of fractional-order parameters on the ECSR, we selected a set of basic parameters as $m=5$, $k=45, \alpha_{1}=0.1, \varepsilon=0.01, K_{1}=0.1$, and $p=0.5$ to analyze the system. According to (32), we can obtain the effect of fractional-order parameters on the ECSR as shown in Figure 1.

Based on Figure 1(a) and comparing the ECSR for integerorder VDP oscillator $\left(K_{1}=0\right)$ with that for fractional-order VDP oscillator, we can know that the existence ranges of the excitation amplitude and frequency will be narrowed along with the increase of the fractional coefficient $K_{1}$. Different from the ECSR for traditional integer-order system, the existence range of ECSR for fractional-order system will not be symmetric about the original frequency value $\sigma=0$ and shift to high-frequency range. From the effects of fractionalorder parameters on the ELDC and ELSC, we could know that the fractional coefficient $K_{1}$ affected the dynamical characteristics of system. Through the analysis of Figure 1(a), one could know that $K_{1}$ enables to narrow the ranges of the excitation amplitude and frequency by the form of ELDC and ELSC and enables to shift the ECSR to high-frequency range by the form of ELSC.

Similarly, the effects of the fractional order $p$ on the ECSR could be found, which is shown in Figure 1(b). According to the effects of the fractional order $p$ on the ELDC, the ranges of the excitation amplitude and frequency are narrowed along with the increase of the fractional order $p$. It is more

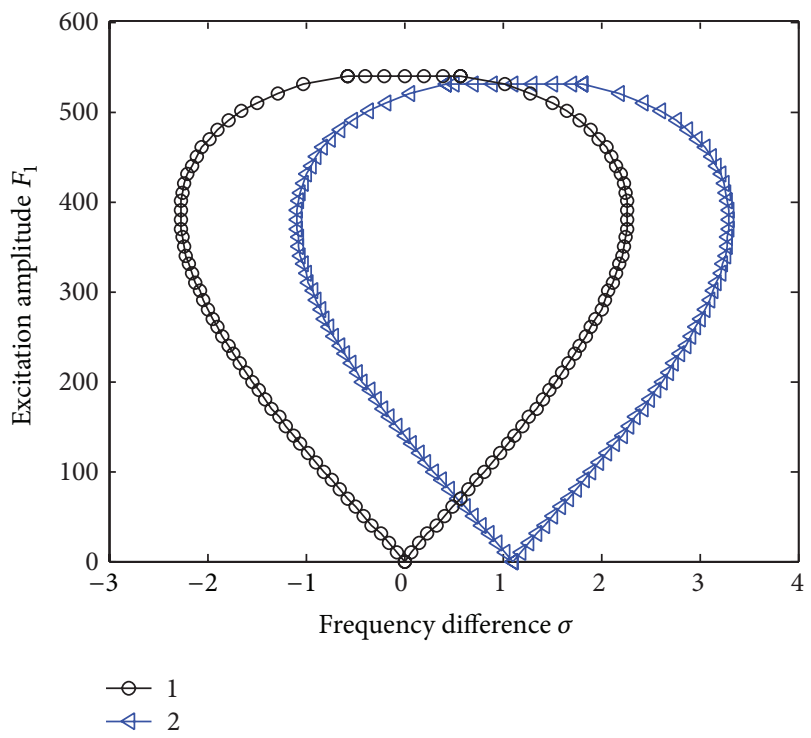

Figure 2: The comparison of the existence condition for subharmonic resonance of the integer-order VDP oscillator $\left(1: K_{1}=0\right)$ with that of the fractional-order counterpart $\left(2: K_{1}=0.05, p=0.1\right)$.

noteworthy that the effects of the fractional order $p$ on the ECSR are fulfilled by the form of ELSC. The ECSR is reduced and shifted right-and-left along the excitation frequency under the control of the ELSC. When $0<p<p_{c}$, the ECSR is shifted to high-frequency range along with the increase of $p$. On the contrary, the ECSR will be shifted to low-frequency range along with the increase of $p$ if $p_{c}<p<1$. 


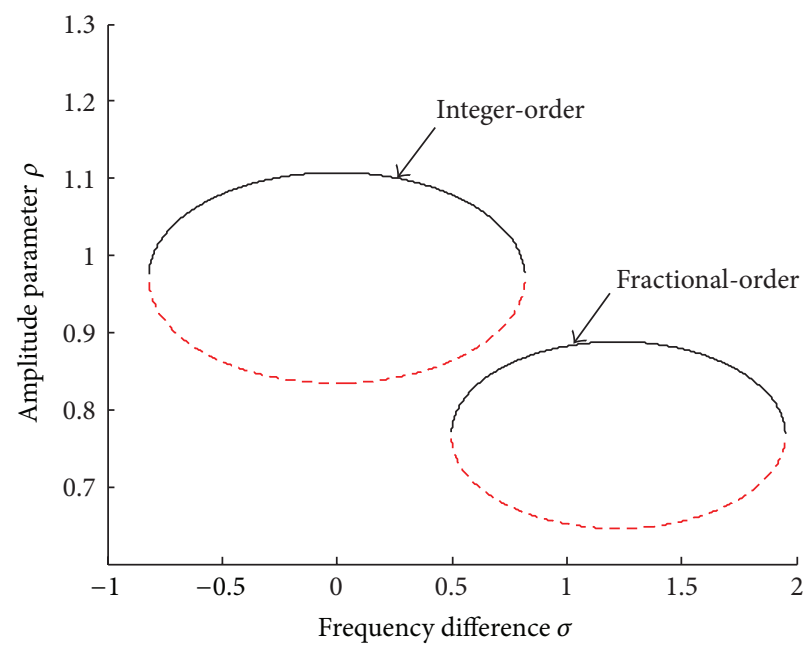

(a) $F_{1}=100$

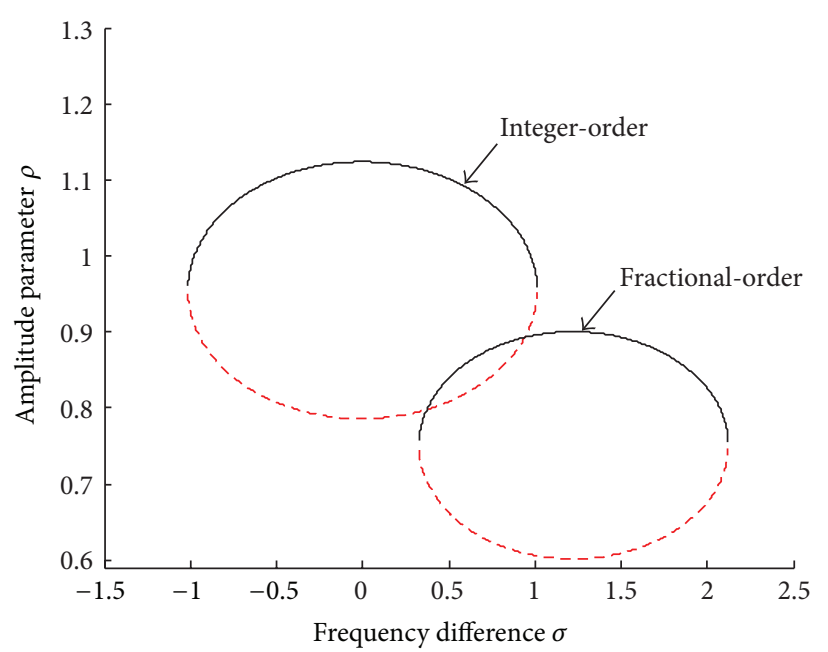

(b) $F_{1}=125$

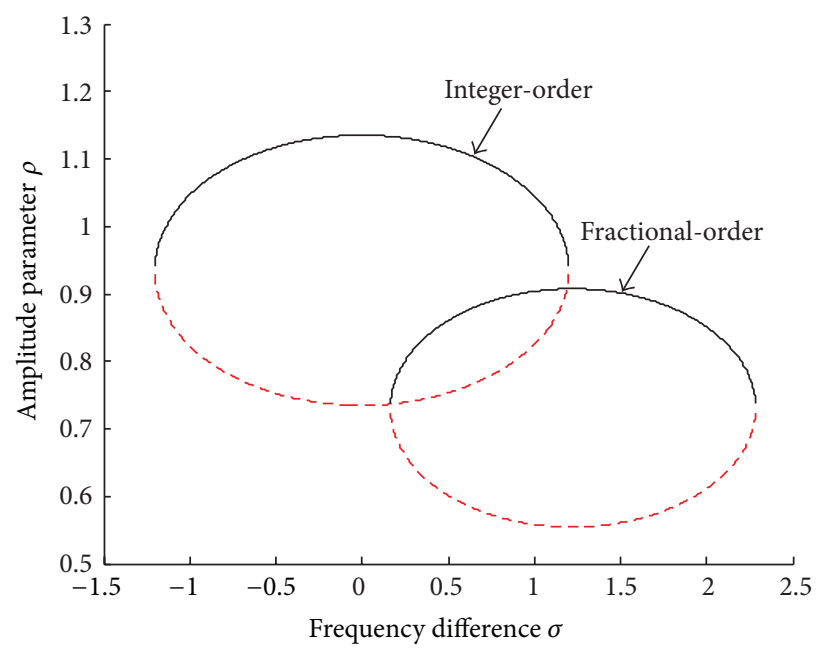

(c) $F_{1}=150$

FIGURE 3: The comparisons of the amplitude-frequency curves of the integer-order VDP oscillator $\left(K_{1}=0\right)$ with that of the fractional-order $\left(K_{1}=0.05, p=0.5\right)$ counterpart in three response modes.

By the above analysis, we get to know that the smaller the fractional-order parameters are, the wider the ranges of the excitation amplitude and frequency are. $K_{1}$ and $p$ also enable to shift the ECSR to the designated direction if they are appropriately selected.

4.2. The Comparison between the Integer-Order and Fractional-Order VDP oscillator. A typical ECSR diagram for fractional-order VDP oscillator $\left(K_{1}=0.05, p=0.1\right)$ associated with the corresponding integer-order counterpart is shown in Figure 2. From Figure 2, we can know that the fractional-order parameters will affect the excitation amplitude and frequency of the ECSR by the form of the ELDC and ELSC. According to (32), the ECSR will have wider ranges of the excitation amplitude and frequency if the damping and stiffness are smaller. Different from the ECSR of traditional integer-order VDP oscillator, the fractional-order parameters also enable to shift the ECSR along excitation frequency by the form of ELSC. From the effects of fractional-order parameters on the ECSR, we also know that the smaller fractional-order parameters are the smaller ECSR shifts to high-frequency range under the control of the ELSC.

In order to illustrate the effects of fractional-order parameters on the dynamical characteristics of VDP oscillator, we compare the amplitude-frequency curves of the integer-order and fractional-order $\left(K_{1}=0.05, p=0.5\right)$ VDP oscillator. The results are shown in Figure 3, where the solid line is for stable solution and the dot line is for unstable one. According to the effects of fractional-order parameters on the ECSR, we analyze three typical amplitude-frequency curves for $F_{1}=100$, 125, and 150, respectively. From the observation of Figure 3, we can know that the response amplitudes of fractional-order system are decreased although the topological structures of amplitude-frequency curves are not changed. Meanwhile, the ranges of the excitation amplitude and frequency are 


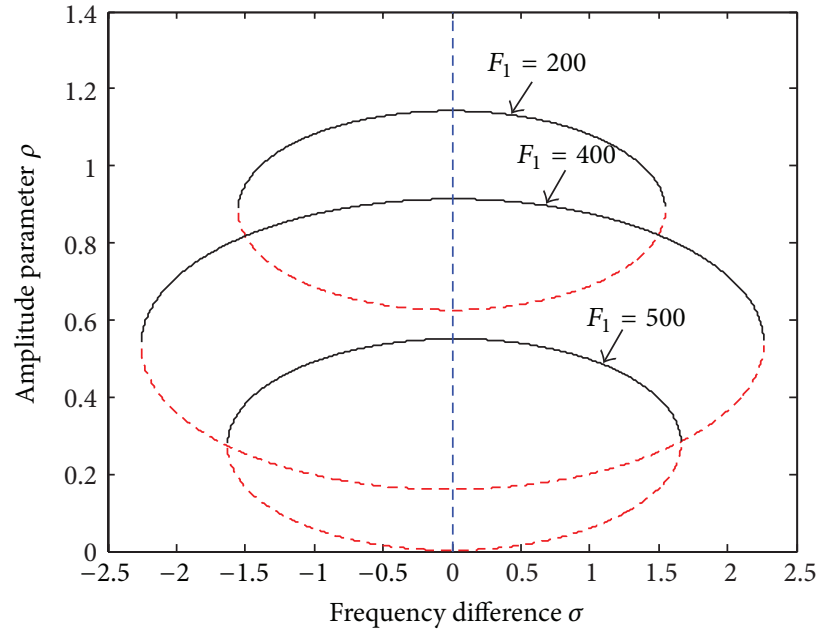

(a)

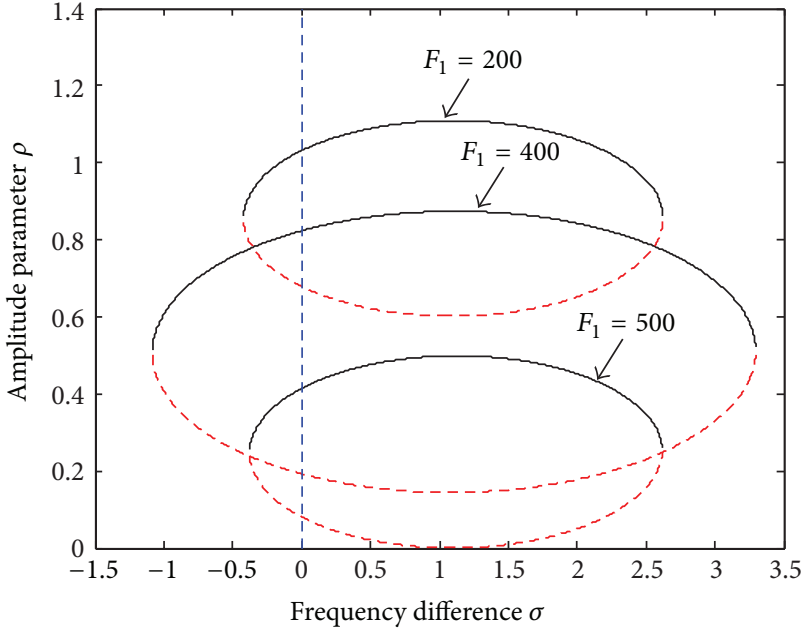

(b)

FIgURE 4: The comparisons of the amplitude-frequency curves of the integer-order VDP oscillator with that of the fractional-order $\left(K_{1}=0.05\right.$, $p=0.1$ ) counterpart: (a) integer-order VDP oscillator; (b) fractional-order VDP oscillator.

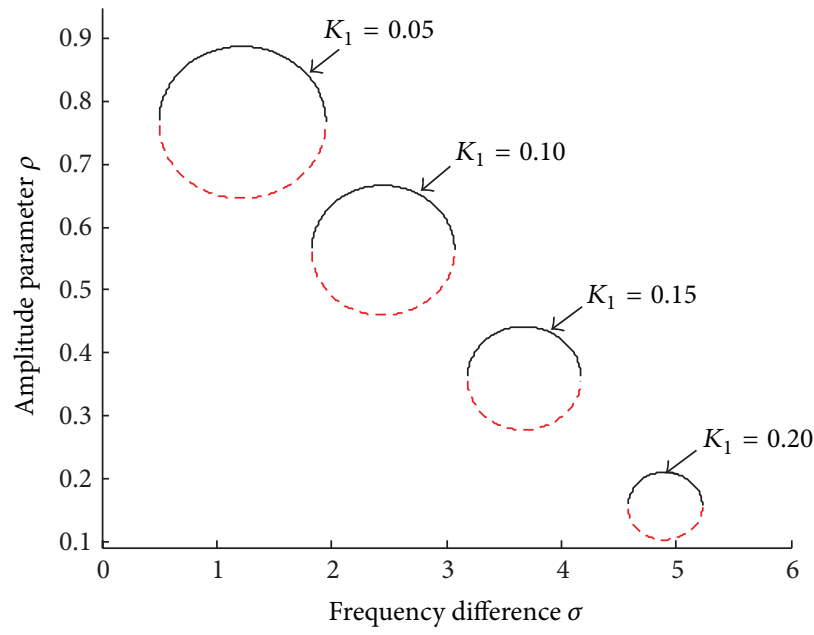

(a) $F_{1}=100$

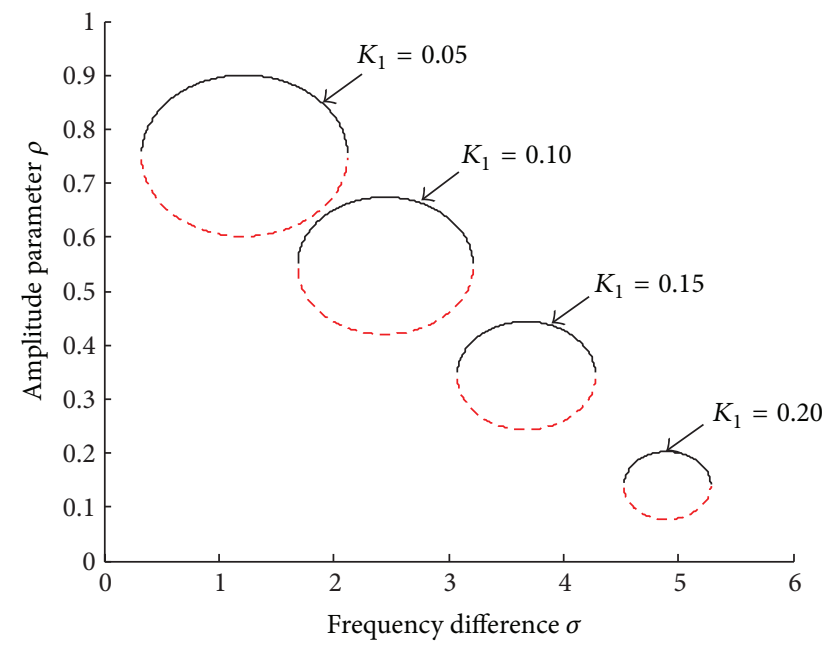

(b) $F_{1}=125$

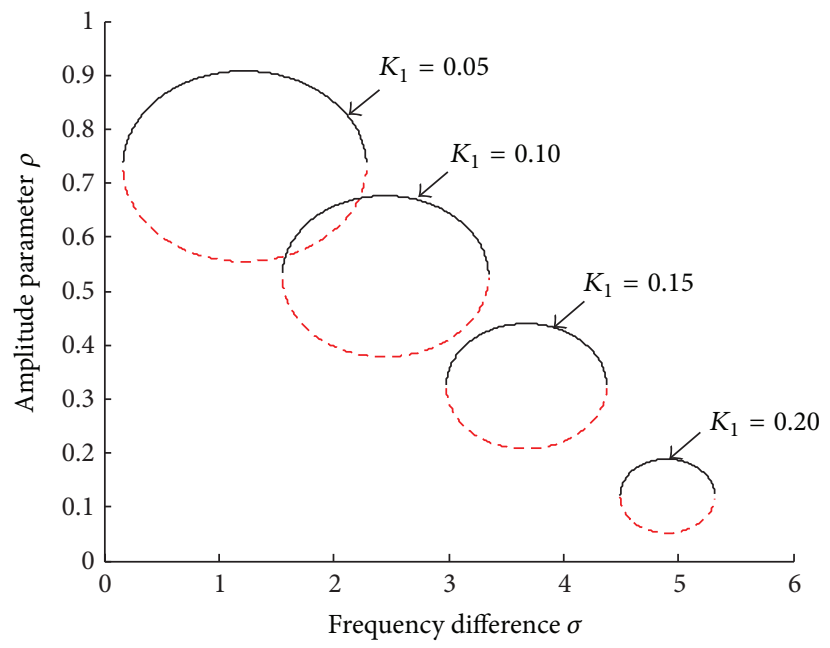

(c) $F_{1}=150$

FIGURE 5: Effects of the fractional coefficient $K_{1}$ on the amplitude-frequency curves in three response modes. 


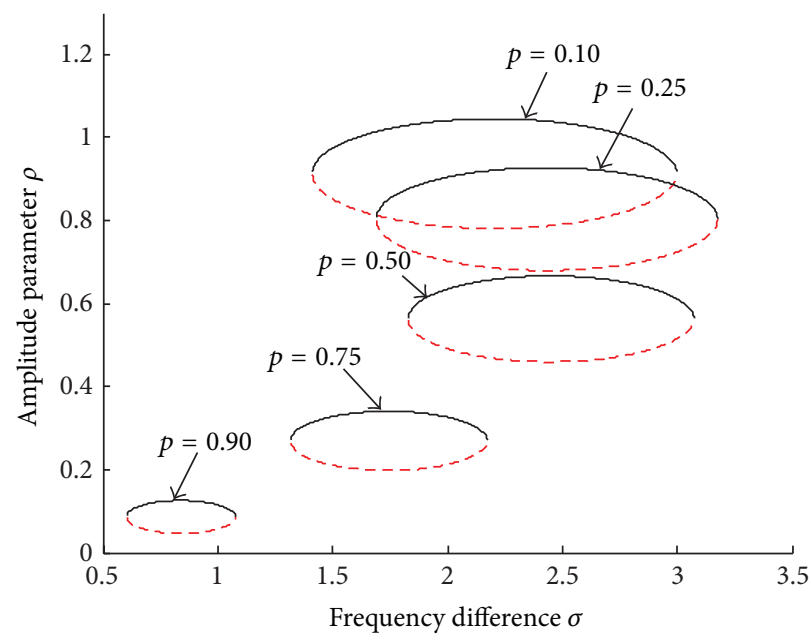

(a) $F_{1}=100$

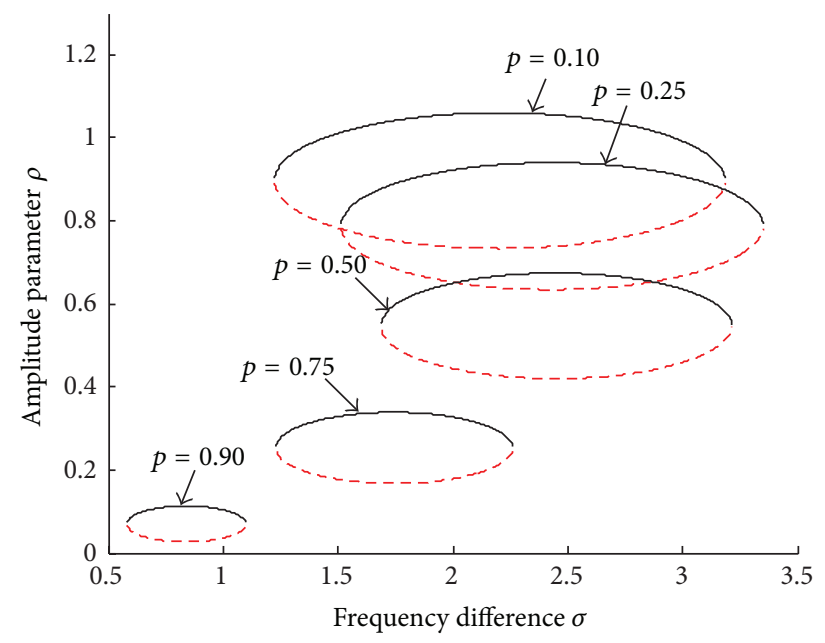

(b) $F_{1}=125$

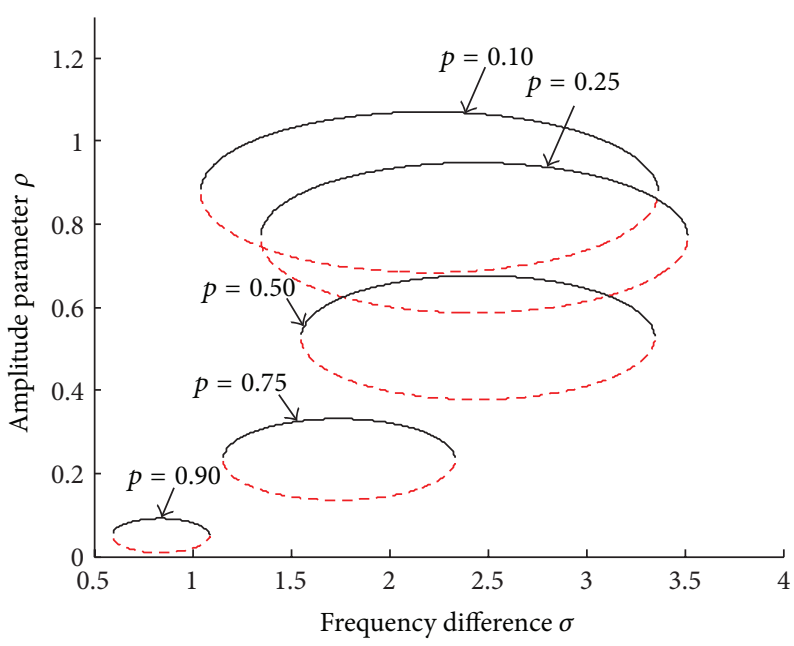

(c) $F_{1}=150$

FIgURE 6: Effects of the fractional order $p$ on the amplitude-frequency curves in three response modes.

narrowed, and the amplitude-frequency curves of fractionalorder system are shifted to high-frequency range. Considering the effects of fractional-order parameters on the ECSR, we can conclude that the fractional-order parameters could decrease the response amplitude by the form of ELDC, shift the amplitude-frequency curve along excitation frequency by the form of ELSC, and affect the range of the excitation frequency by the form of ELDC and ELSC simultaneously.

The ranges of the excitation amplitude and frequency are wider when fractional-order parameters are smaller. According to the observation of Figure 2, we can compare the relative topological structures of three typical amplitudefrequency curves for $F_{1}=200,400$, and 500 as shown in Figure 4. From Figure 4, it could be found that the relative topological structures of amplitude-frequency curve for fractional-order system are not changed. The effects of fractional-order parameters on the amplitude-frequency curves in Figure 4 are similar to the corresponding ones of Figure 3.
Through the above analysis, one could find that the fractional-order parameters will decrease the response amplitude by the form of ELDC, shift the amplitude-frequency curve along excitation frequency by the form of ELSC, and affect the existence ranges of excitation frequency by the form of ELDC and ELSC. But they could not affect the topological structure of amplitude-frequency curve for subharmonic resonance. Therefore, the dynamical characteristics of the original integer-order system could be optimized by introducing appropriate fractional-order term.

4.3. Effects of Fractional-Order Parameters on the AmplitudeFrequency Curves. Based on the comparison of the amplitude-frequency curves between the integer-order and fractional-order system, we study the effects of fractionalorder parameters on the amplitude-frequency curves so as to optimize the system by choosing appropriate fractional-order parameters. 


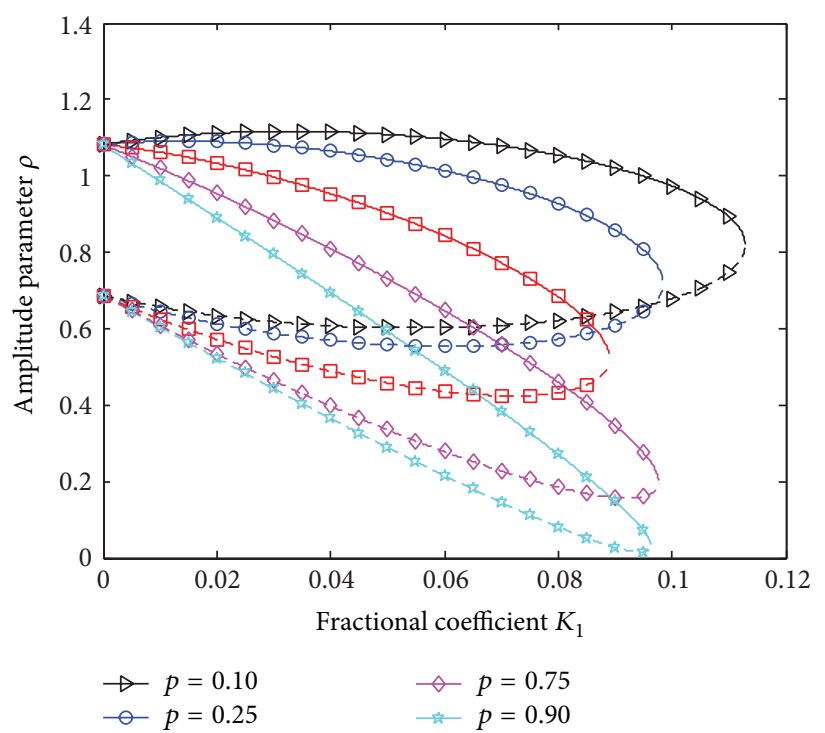

(a) $F_{1}=200$

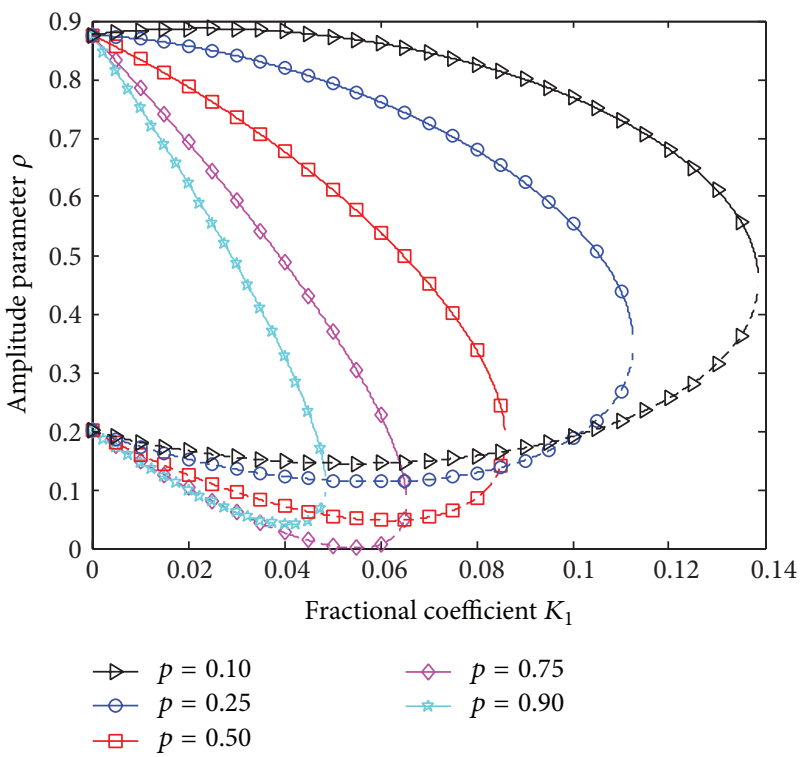

(b) $F_{1}=400$

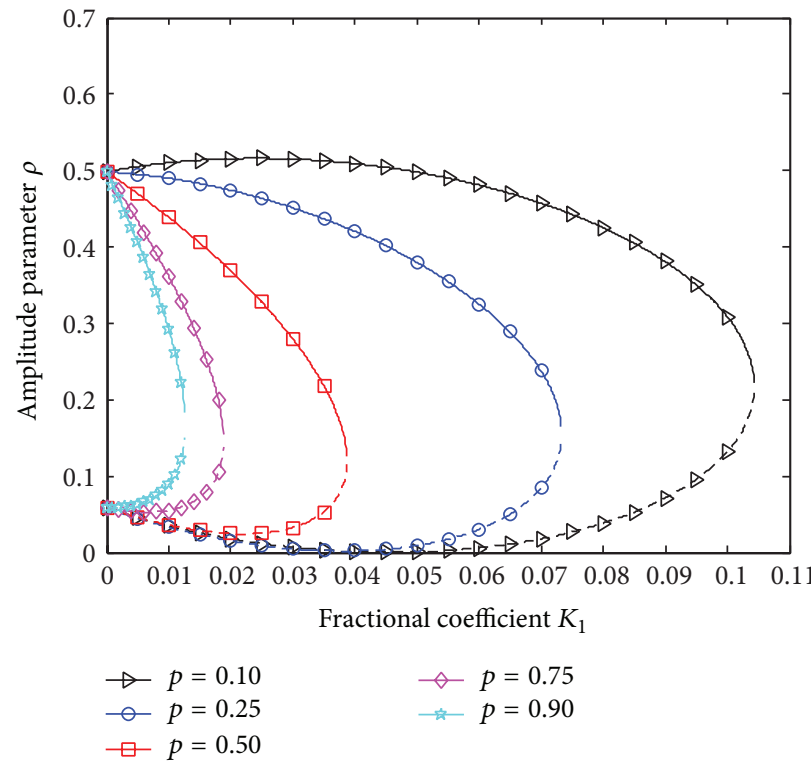

(c) $F_{1}=500$

FIGURE 7: Effects of the fractional coefficient $K_{1}$ on the response amplitudes in three response modes for $\sigma=1$.

One could obtain the different amplitude-frequency curves in the three typical modes shown in Figure 5, where $p=0.5$ and the fractional coefficient $K_{1}$ is changed. Through the analysis of Figure 5, we could find that the fractional coefficient $K_{1}$ will affect the amplitude of the amplitudefrequency curve by the form of ELDC, shift the amplitudefrequency curve along excitation frequency by the form of ELSC, and affect the existence ranges of excitation frequency by the form of ELDC and ELSC. It means that the response amplitude will be decreased, the existence ranges of excitation frequency will be narrowed, and the amplitude-frequency curves will be gradually shifted to high-frequency range along with the increase of $K_{1}$.
When the fractional order $p$ is changed, we get three kinds of amplitude-frequency curves shown in Figure 6, where $K_{1}=0.1$. Similar to the analysis of Figure 5, it could be found that the response amplitude and the existence ranges of excitation frequency are decreased along with the increase of $p$. Based on the effects of the fractional order $p$ on the ELSC, we could know that the amplitude-frequency curves will be shifted to high-frequency range along with the increase of $p$ when $0<p<p_{c}$, and the corresponding ones will be shifted to low-frequency range with the increase of $p$ if $p_{c}<p<1$.

Based on the effects of fractional-order parameters on the amplitude-frequency curves for subharmonic resonance of VDP oscillator, one could find that the response amplitude 


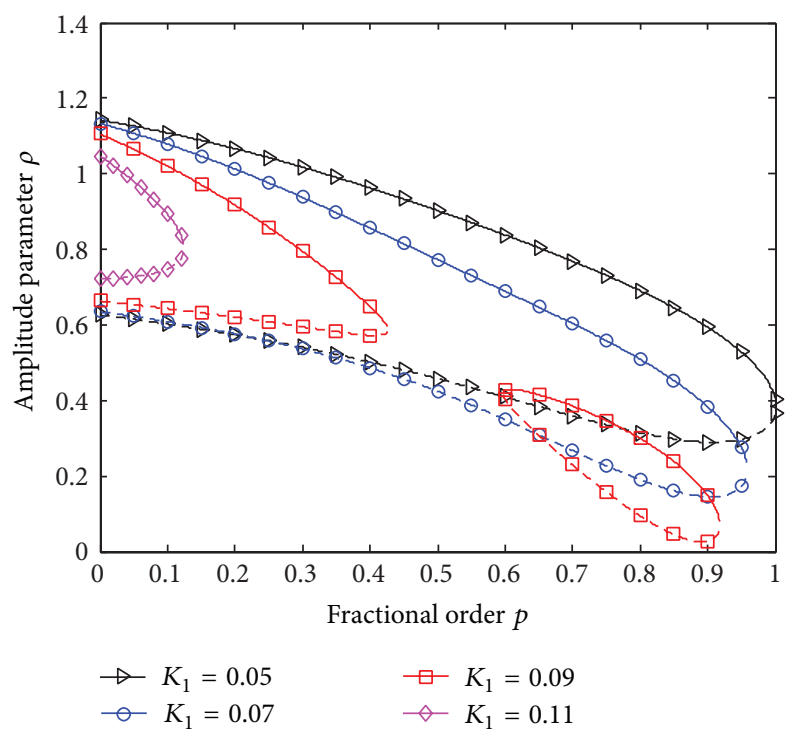

(a) $F_{1}=200$

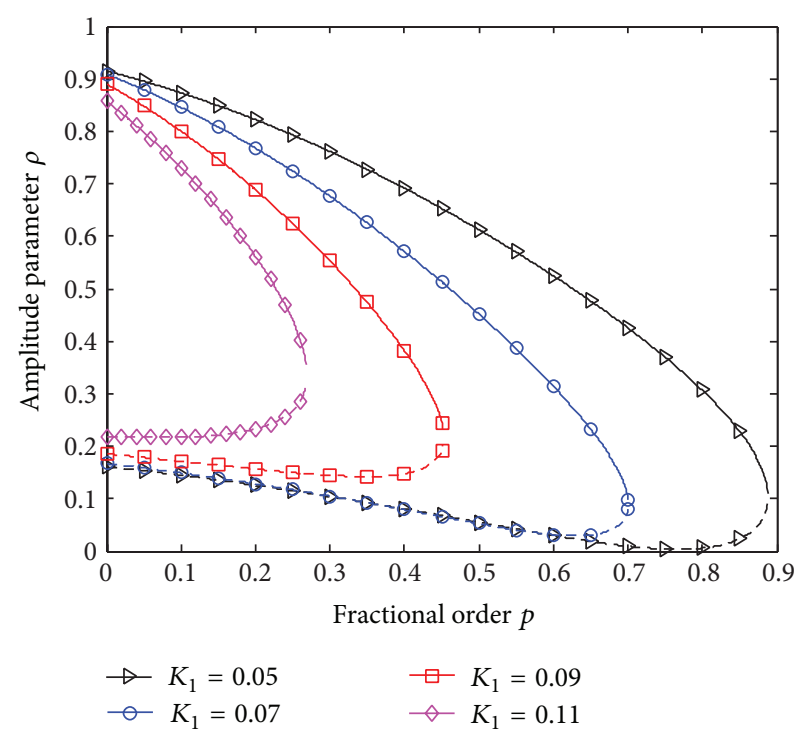

(b) $F_{1}=400$

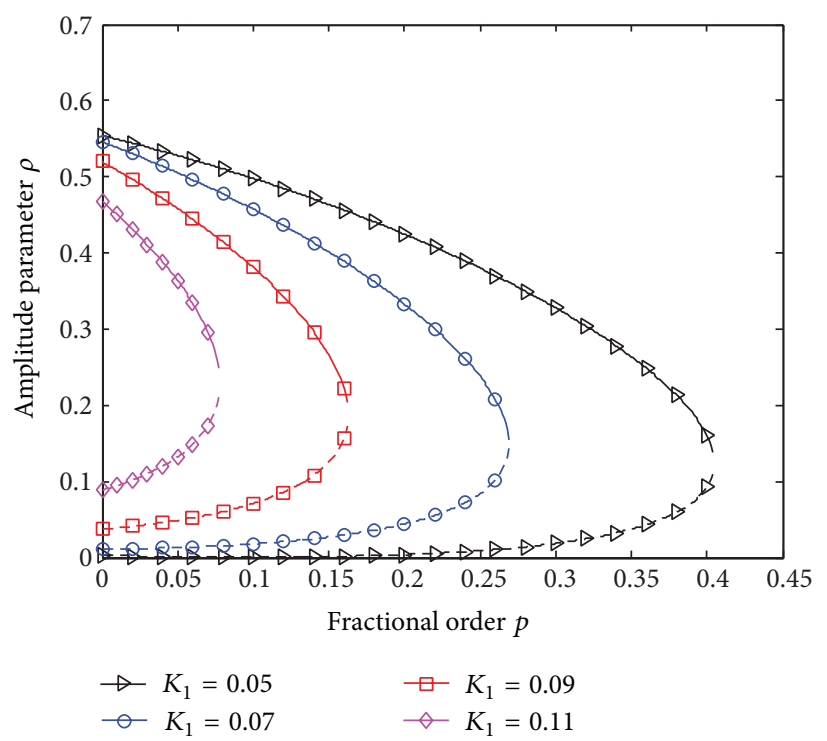

(c) $F_{1}=500$

FIGURE 8: Effects of the fractional order $p$ on the response amplitudes in three response modes for $\sigma=1$.

and existence range of excitation frequency for amplitudefrequency curve are decreased along with the increase of the fractional-order parameters. When $K_{1}$ and $p$ are smaller, one could obtain the amplitude-frequency curve whose response amplitude and existence range of excitation frequency are similar to the corresponding ones in the integer-order system. When $K_{1}$ and $p$ are larger, we can find that the fractionalorder term could greatly change the dynamical characteristics of the original one. Therefore, we can choose the appropriate fractional-order parameters to optimize the system based on those results.

4.4. Effects of Fractional-Order Parameters on the Response Amplitude. As an important dynamical characteristic, the response amplitude generally reflects the system energy and has significant value. It is meaningful to study the effects of fractional-order parameters on system response amplitude, which is beneficial to improve the stability of response amplitude.

The effects of the fractional coefficient $K_{1}$ on the response amplitude for $F_{1}=200,400$, and 500 are shown in Figure 7, where the solid line is for stable response amplitude and the dot line is for the unstable one. From the observation of Figure 7, we could know that $K_{1}$ will directly affect the response amplitude for frequency value $\sigma=1$ by the form of ELDC and indirectly affect the corresponding one by the form of ELSC when $K_{1}$ is gradually increased. Moreover, based on the characteristics of amplitude-frequency curve 


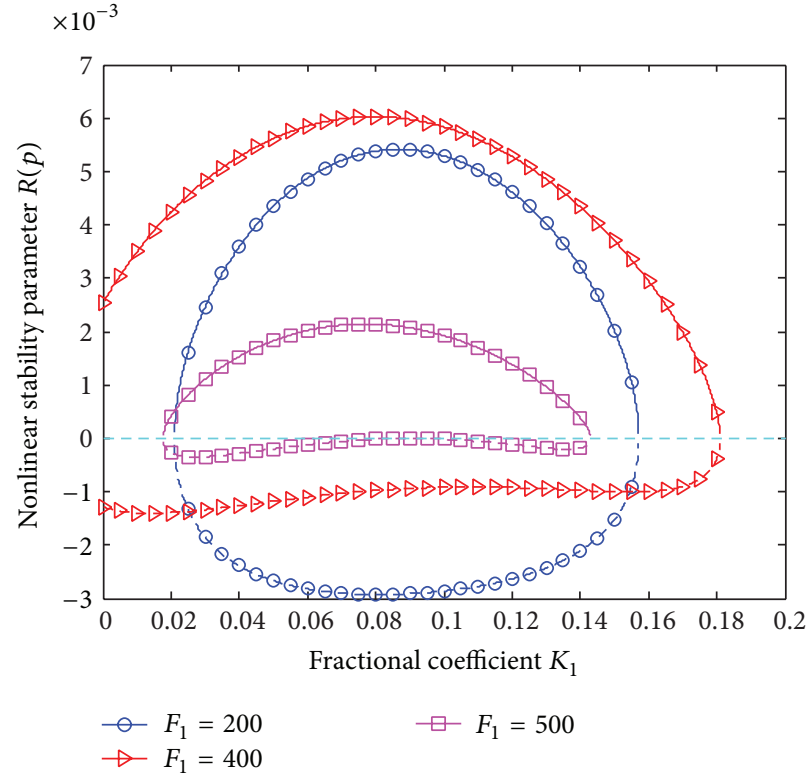

(a) Nonlinear stability parameter

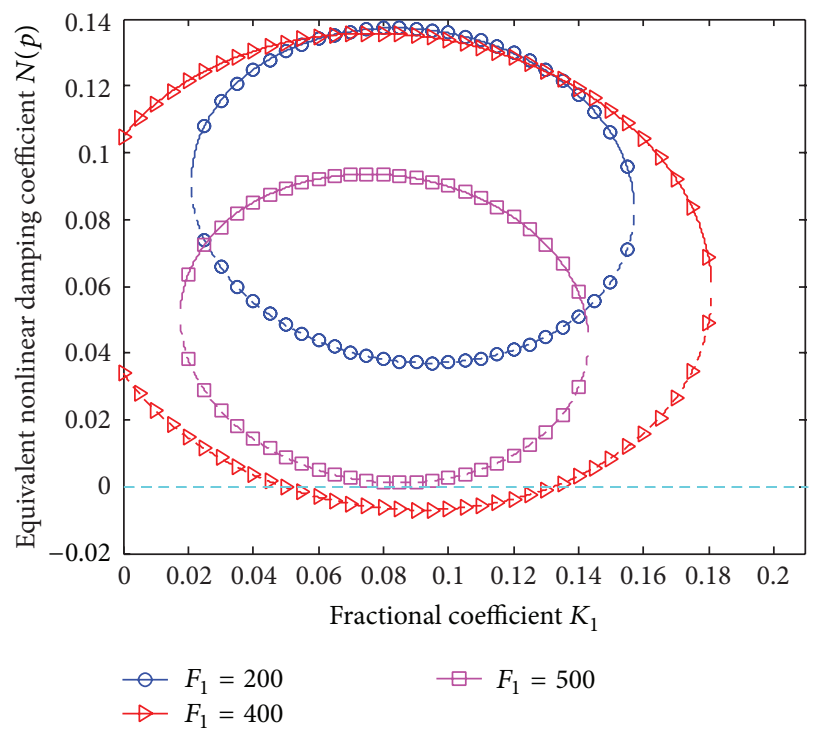

(b) Equivalent nonlinear damping coefficient

FIGURE 9: Effects of the fractional coefficient $K_{1}$ on system stability for $\sigma=2$ and $p=0.1$.

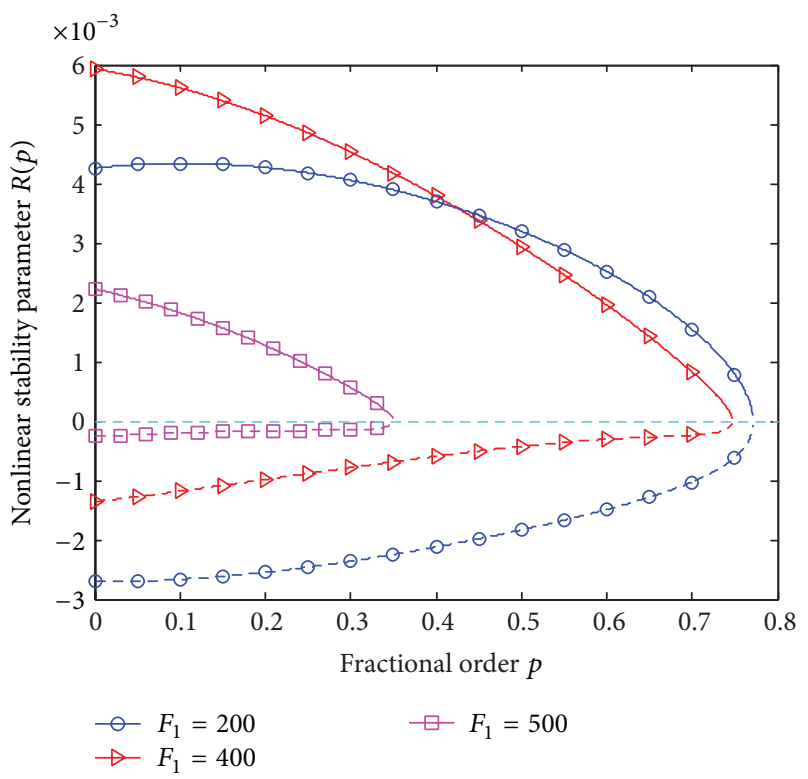

(a) Nonlinear stability parameter

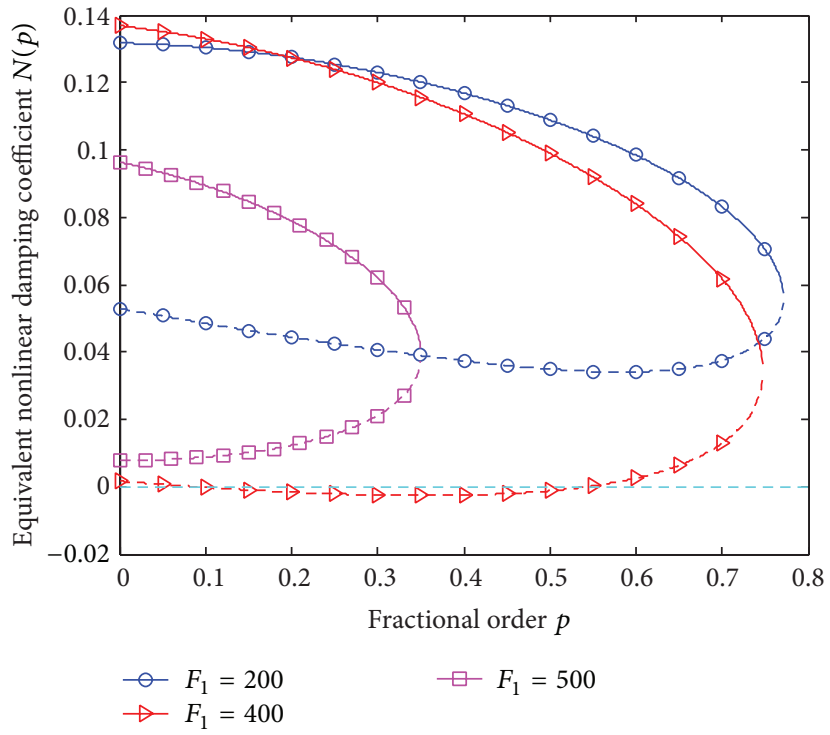

(b) Equivalent nonlinear damping coefficient

Figure 10: Effects of the fractional order $p$ on system stability for $\sigma=2$ and $K_{1}=0.05$.

for subharmonic resonance of VDP oscillator, one could know that any stable amplitude must be corresponding to an unstable one, and the unstable one is smaller than the stable one. It means that the change of the fractional coefficient $K_{1}$ will change both the system stability and the response amplitude. We could also know from Figure 7 that the bigger the fractional order $p$ is, the smaller the response amplitude at $\sigma=1$ becomes. It is also worth noting that the value ranges of the response amplitude about the change of $K_{1}$ are not gradually decreasing along with the increase of $p$ as shown in Figure 7(a). Based on the characteristics of the ECSR, we known that $K_{1}$ and $p$ will directly affect the response amplitude for a particular frequency difference $\sigma$ by the form of ELDC and also indirectly affect the corresponding one by the form of ELSC through shifting the corresponding amplitude-frequency curve. One had known from (19a) and (19b) that the amplitude-frequency curve will shift to highfrequency range along with the increase of $p$ when $0<p<p_{c}$ 


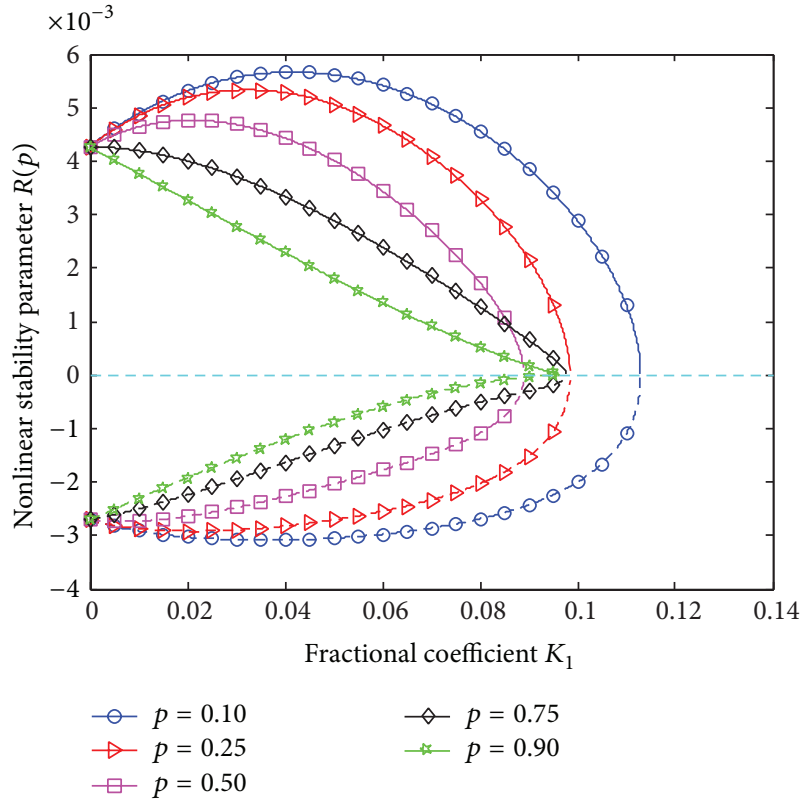

(a) Effect of $K_{1}$ on $R(p)$

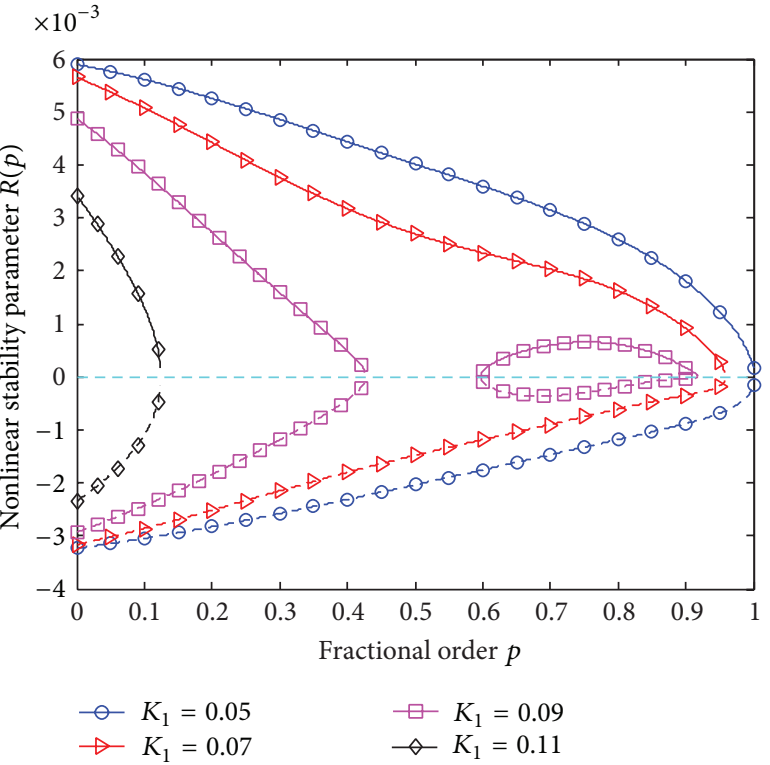

(c) Effect of $p$ on $R(p)$

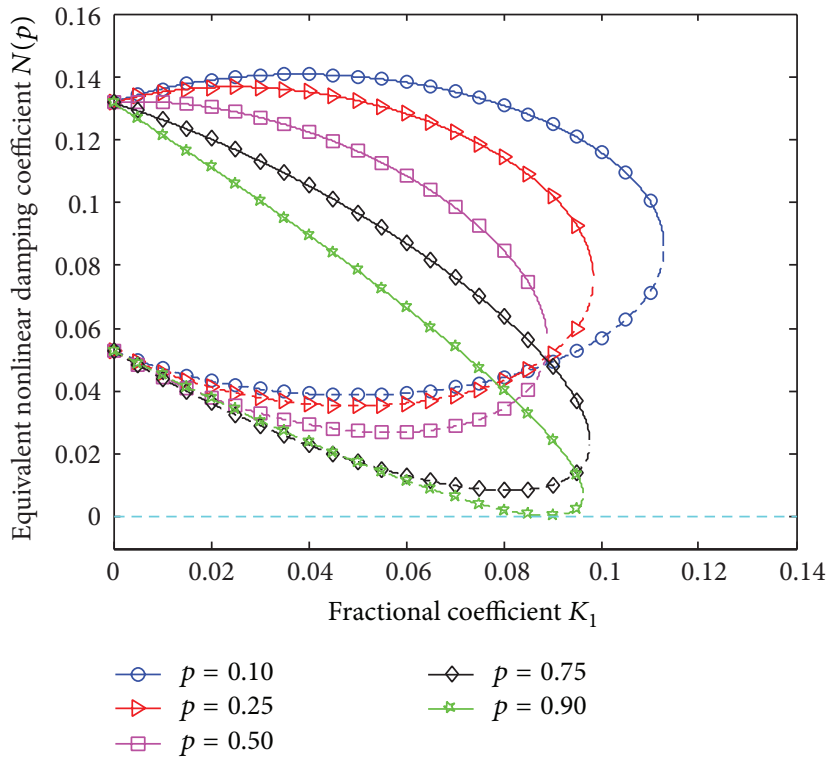

(b) Effect of $K_{1}$ on $N(p)$

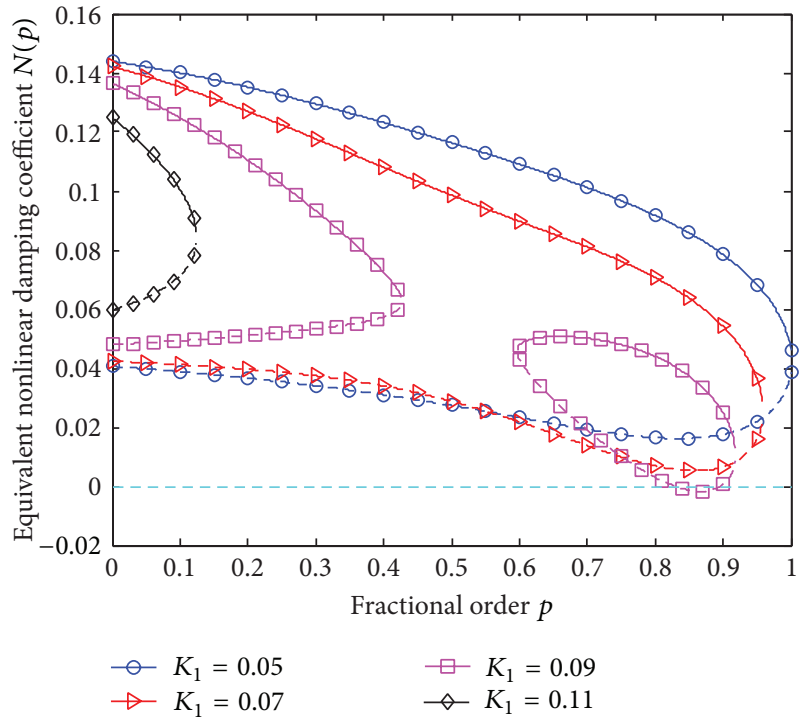

(d) Effect of $p$ on $N(p)$

FIGURE 11: Effects of the fractional-order parameters on system stability for $\sigma=1$ and $F_{1}=200$.

and shift to low-frequency range along with the increase of $p$ if $p_{c}<p<1$. By combining all the above analysis, one could conclude that it maybe generate the phenomenon for Figure 7(a) under the effects of ELDC and ELSC on response amplitude. for Figure 7(a) under the effects of ELDC and ELSC on response amplitude.

The effects of the fractional order $p$ on the response amplitude for $F_{1}=200,400$, and 500 are shown in Figure 8 . It is similar to the analysis of Figure 7 , one could know that the response amplitude at $\sigma=1$ will be decreased along with the increase of fractional-order parameters. It is worth noting that there is a segmented phenomenon for $K_{1}=$
0.09 in Figure 8(a). From the effects of $K_{1}$ on the response amplitude, we could know that the response amplitude for a particular frequency difference $\sigma$ will be affected by $K_{1}$ and $p$ simultaneously through the way of ELDC and ELSC. As to the effects of $p$ on the response amplitude, one could find that the amplitude-frequency curves will shift to high-frequency range and then shift to low-frequency in the procedure of $p$ from 0 to 1 . And the value of response amplitude will be increased and then decreased in the same procedure.

Through the analysis of the effects of fractional-order parameters on the response amplitude, we could find that one can effectively control the value of stable response amplitude 


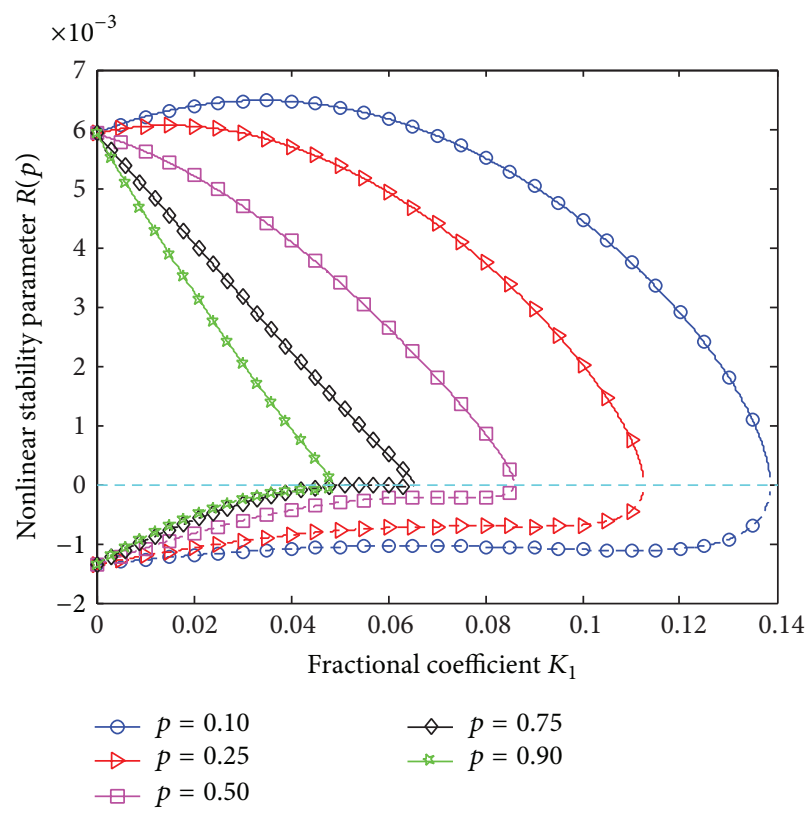

(a) Effect of $K_{1}$ on $R(p)$

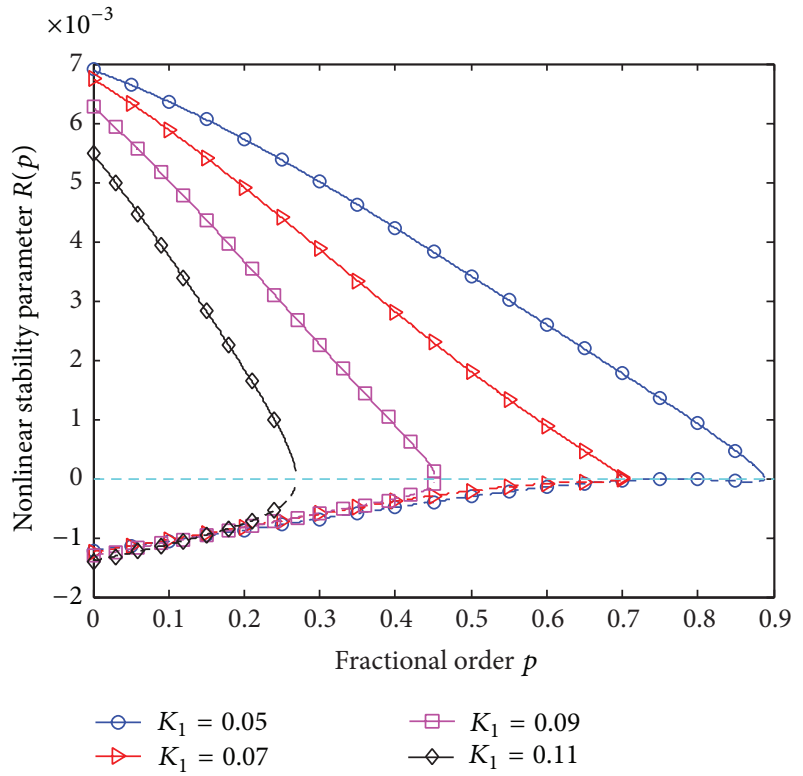

(c) Effect of $p$ on $R(p)$

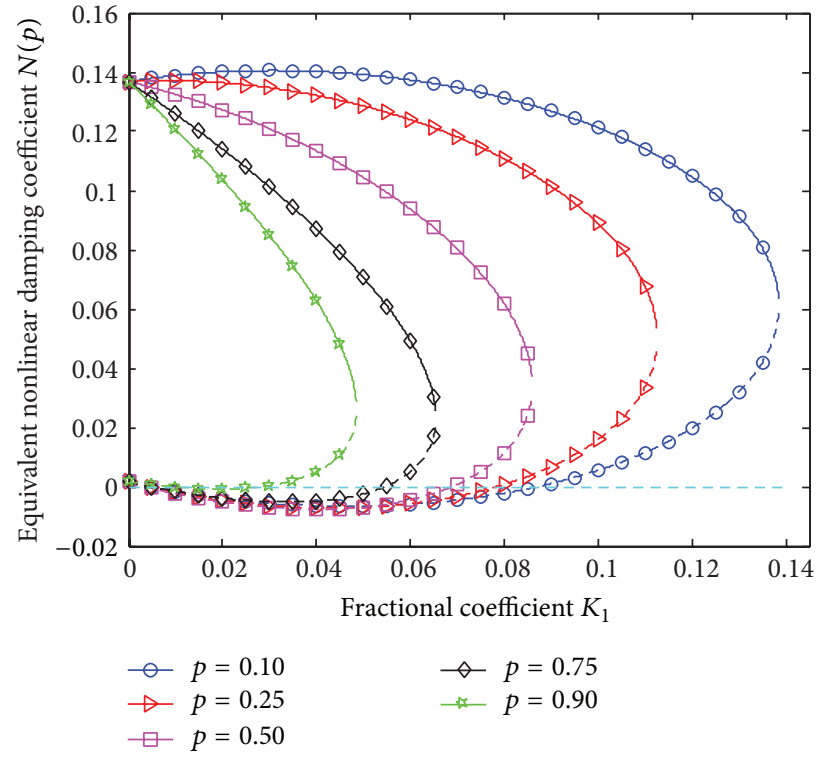

(b) Effect of $K_{1}$ on $N(p)$

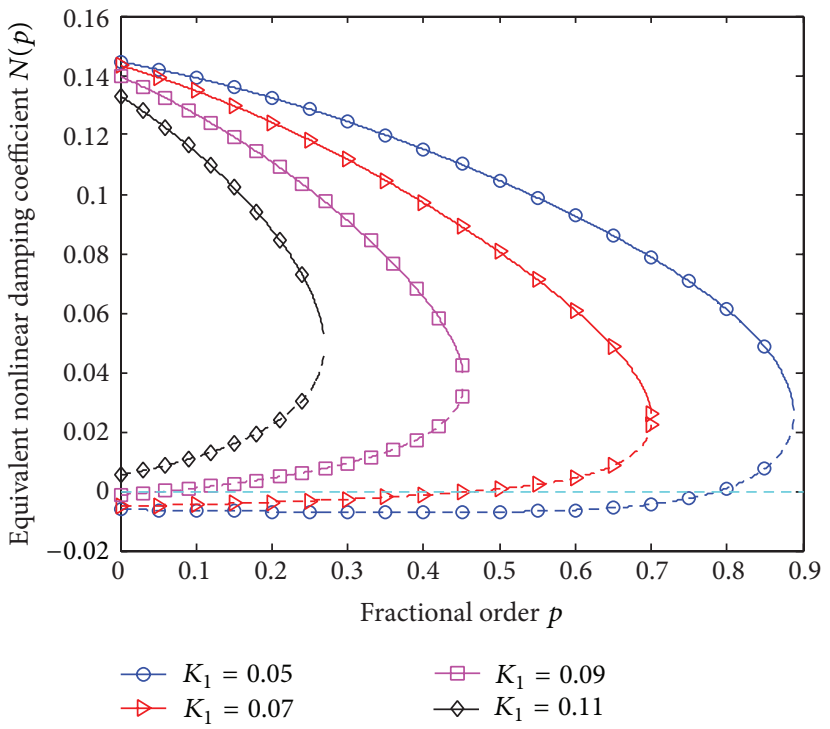

(d) Effect of $p$ on $N(p)$

FIGURE 12: Effects of the fractional-order parameters on system stability for $\sigma=1$ and $F_{1}=400$.

by changing fractional-order parameters. We also find that the larger the excitation amplitude is, the simpler the effects of fractional-order parameters on the response amplitude are. It is meaningful for optimizing system response amplitude.

\subsection{Effects of Fractional-Order Parameters on the System Stability}

4.5.1. Effects of Excitation Amplitude on the Relative Stability of the Stable Solution. The stability of steady-state solution is particularly important. The effects of the fractional coefficient $K_{1}$ on the system stability are shown in Figure 9, where the solid lines satisfy the stability condition and the dot lines do not. Based on the stability conditions for the steady-state solution of (38a) and (38b), we could find that the solution is stable only if the ENDC and NSP are positive simultaneously. We get to know from Figure 9 that the unstable solutions correspond to $N(p)>0, R(p)<0$ for $F_{1}=200$ and 500 , and it can make the system generate the unstable periodic vibration. For $F_{1}=400$, the unstable solutions correspond to two cases, that is $N(p)>0, R(p)<0$ and $N(p)<0, R(p)<0$. 


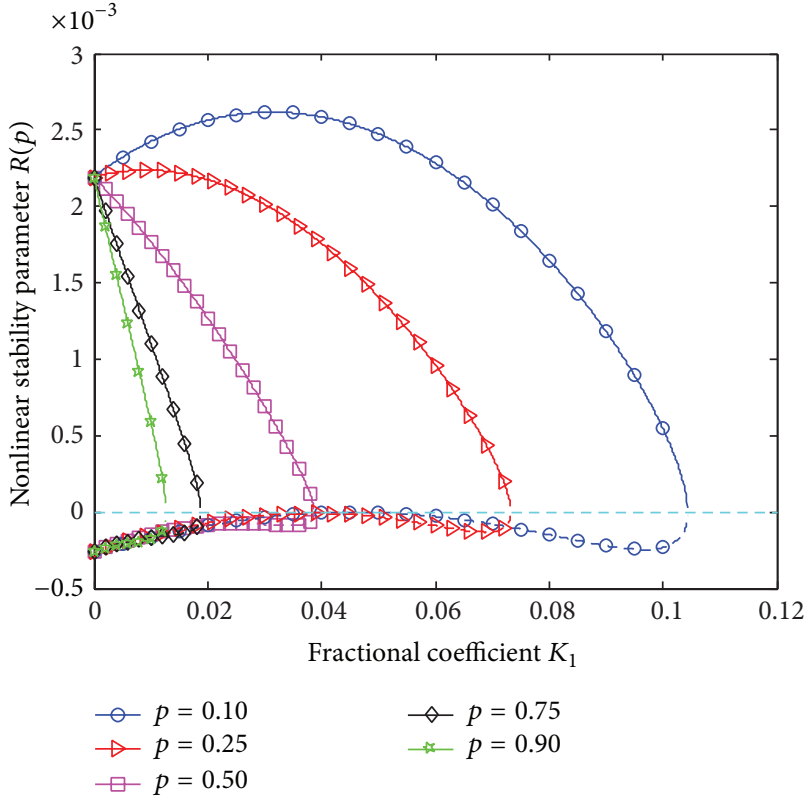

(a) Effect of $K_{1}$ on $R(p)$

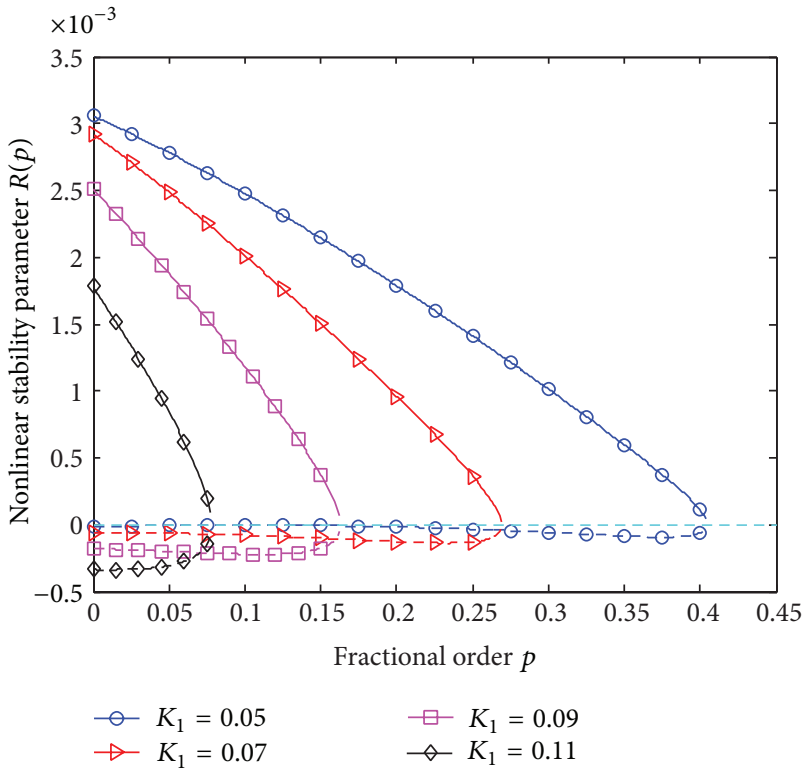

(c) Effect of $p$ on $R(p)$

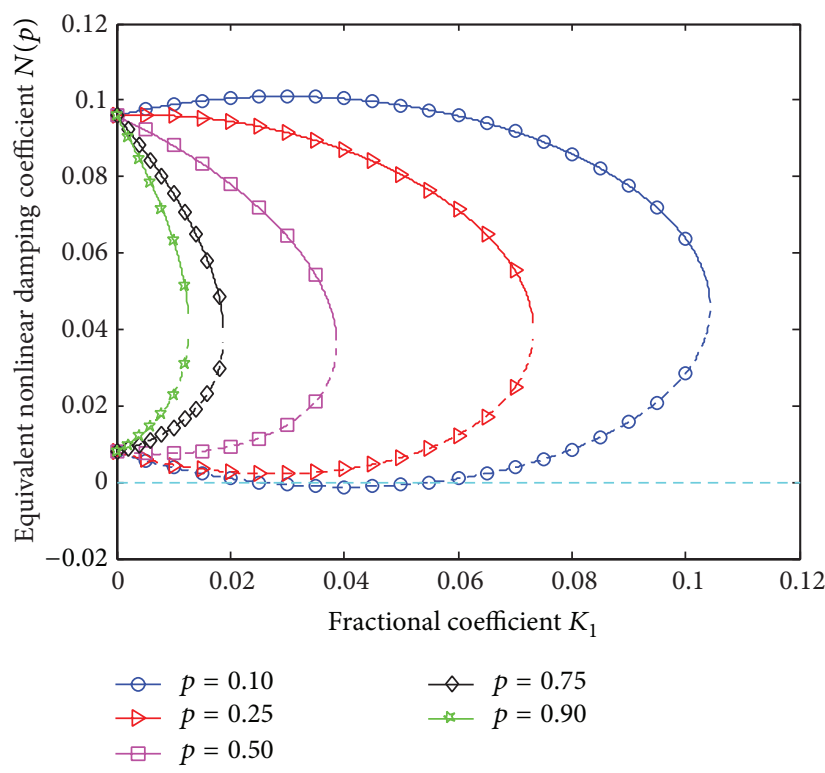

(b) Effect of $K_{1}$ on $N(p)$

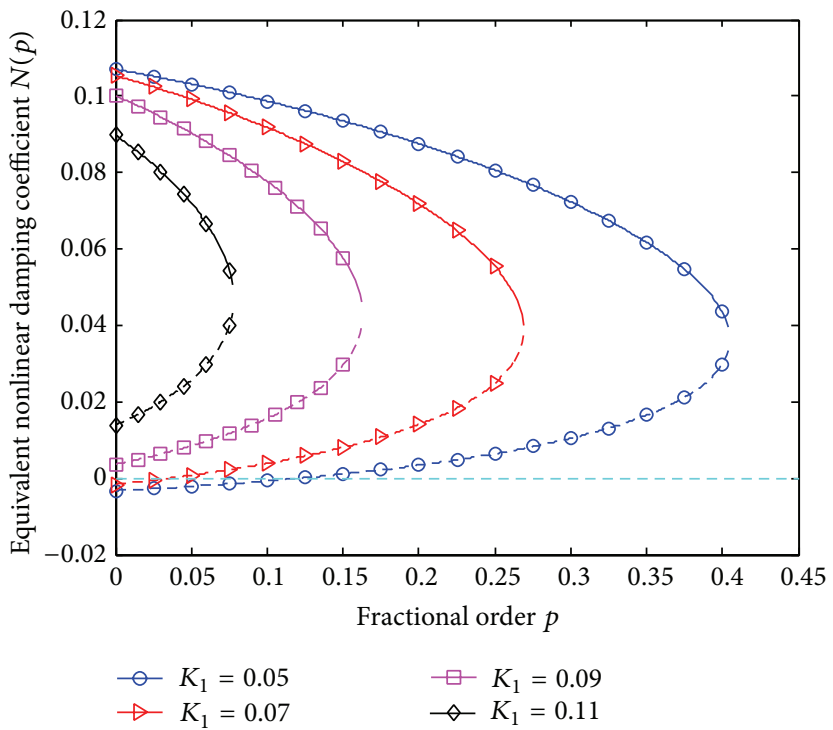

(d) Effect of $p$ on $N(p)$

FIGURE 13: Effects of the fractional-order parameters on system stability for $\sigma=1$ and $F_{1}=500$.

According to the extent of the stable solution curves away from zero axis, we can judge the relative stability of solutions. From the observation of Figure 9, we find that the relative stability of the stable solutions for $F_{1}=400$ is better than the corresponding ones for others. Through analysis, one could know that the value range of the excitation frequency for $F_{1}=400$ is wider, so that it can withstand wider variation range of fractional-order parameters. Therefore, the relative stability of the stable solutions for $F_{1}=400$ is better for a particular frequency difference $\sigma$.

The effects of the fractional order $p$ on the system stability are shown in Figure 10. Similar to the analysis of Figure 9, we get to know that the unstable solutions correspond to $N(p)>$ $0, R(p)<0$ for $F_{1}=200$ and 500 , and the unstable solution for $F_{1}=400$ corresponds to $N(p)>0, R(p)<0$ and $N(p)<$ $0, R(p)<0$. We could also know from Figure $10(\mathrm{~b})$ that the relative stability of the stable solutions for $F_{1}=400$ is better when $p<0.2$, and the relative stability of the corresponding ones for $F_{1}=200$ is better when $p>0.4$, from Figure 10(a). Based on the characteristics of the ECSR, we know that the smaller the excitation amplitude $F_{1}$ is, the easier to satisfy the ECSR the system is. From the above analysis, one could know that the relative stability of the stable solutions is better when $F_{1}$ is smaller and $p$ is larger. 
4.5.2. Effects of Fractional-Order Parameters on the Relative Stability of the Stable Solution. The effects of fractionalorder parameters on the system stability when $K_{1}$ and $p$ are changing simultaneously are shown in Figures 11, 12 and 13. We could know from Figure 11 to Figure 13 that the larger the fractional-order parameters are, the worse the relative stability of system stable solution for a particular frequency difference $\sigma$ is. From the effects of fractional coefficient $K_{1}$ on the system stability, we could know that the relative stability of the stable solution for fractional-order system is better than the corresponding one for integerorder system $\left(K_{1}=0\right)$ when fractional-order parameters are smaller. The relative stability of the stable solution gradually becomes worse along with the increase of $p$. Especially, the relative stability of the stable solution for integer-order system is worse than the corresponding one for fractional-order system when $p \rightarrow 1$. From Figure 11, we also find that the effects of fractional-order parameters on system stability parameters are more complex and there is a segmented phenomenon for $K_{1}=0.09$. Based on the effects of fractional-order parameters on the response amplitude, we could know that the system amplitude-frequency curve is shifted along excitation frequency and the corresponding range of excitation frequency is narrowed when excitation amplitude $F_{1}$ is smaller. At the same time, the similar results could be obtained from Figures 12 and 13. Under the effect of both the offset of amplitude-frequency curve and the change of the range of excitation frequency, the stable solutions may produce segmented phenomenon along with the changes of fractional-order parameters.

According to the effects of fractional-order parameters on system stability parameters, we know that one can obtain the stable periodic solutions whose relative stability is better when $K_{1}$ and $p$ are smaller. We also know that the stable solutions with better relative stability can withstand wider variation ranges of fractional-order parameters. Accordingly, it is important to optimize the system by appropriately changing of the fractional-order parameters.

\section{Conclusions}

The 1/3 subharmonic resonance of fractional-order van der Pol (VDP) oscillator is studied. Through the analysis of the effects of fractional-order parameters on the response amplitude, we found that the fractional-order parameters will affect response amplitude in a certain frequency difference by the form of ELDC and ELSC. Through the analysis of the effects of fractional-order parameters on the amplitudefrequency curves, we get to know that the fractional-order parameters will affect the amplitude of amplitude-frequency curve by the form of ELDC, shift the amplitude-frequency curve along the excitation frequency by the form of ELSC, and affect the ranges of the excitation frequency by the form of ELDC and ELSC simultaneously. Through the analysis of the effects of fractional-order parameters on the ECSR, we find that the smaller the fractional-order parameters are, the wider the ranges of the excitation amplitude and frequency are. Through the analysis of the effects of fractional-order parameters on the stability parameters, we could draw the conclusion that the relative stability of the corresponding stable solution is better when the range of the excitation frequency is wider and the fractional-order parameters are smaller. These results are greatly important in the dynamical analysis on fractional-order system and have important significance for optimizing the system.

\section{Conflict of Interests}

The authors declare that there is no conflict of interests regarding the publication of this paper.

\section{Acknowledgments}

The authors are grateful to the support of the National Natural Science Foundation of China (nos. 11072158 and 11372198), the Program for New Century Excellent Talents in University (NCET-11-0936), and the Cultivation plan for Innovation team and leading talent in Colleges and universities of Hebei Province (LJRC018).

\section{References}

[1] K. B. Oldham, "Fractional differential equations in electrochemistry," Advances in Engineering Software, vol. 41, no. 1, pp. 9-12, 2010.

[2] W. G. Glöckle and T. F. Nonnenmacher, "Fractional integral operators and fox functions in the theory of viscoelasticity," Macromolecules, vol. 24, no. 24, pp. 6426-6434, 1991.

[3] D.-Z. Li, J. Cao, S.-T. Guan, and T.-W. Tan, "Research and implementation of a fractional predictive controller," Control Theory and Applications, vol. 27, no. 5, pp. 658-662, 2010.

[4] Z. Chen, Y. L. Peng, S. W. Wang, and F. L. Yin, "From discrete to continuous-fractional signal processing theories, methods and applications," Acta Electronica Sinica, vol. 40, no. 11, pp. 2282 2289, 2012.

[5] X. Chen, L. Wei, J. Sui, X. Zhang, and L. Zheng, "Solving fractional partial differential equations in fluid mechanics by generalized differential transform method," in Proceedings of the 2nd International Conference on Multimedia Technology (ICMT '11), pp. 2573-2576, July 2011.

[6] R. Metzler and J. Klafter, “The random walk's guide to anomalous diffusion: a fractional dynamics approach," Physics Reports, vol. 339, no. 1, pp. 1-77, 2000.

[7] G. M. Zaslavsky, "Chaos, fractional kinetics, and anomalous transport," Physics Reports, vol. 371, no. 6, pp. 461-580, 2002.

[8] S. Müller, M. Kästner, J. Brummund, and V. Ulbricht, "On the numerical handling of fractional viscoelastic material models in a FE analysis," Computational Mechanics, vol. 51, no. 6, pp. 999-1012, 2013.

[9] Y. J. Shen, S. P. Yang, H. J. Xing, and H. X. Ma, "Primary resonance of Duffing oscillator with two kinds of fractional-order derivatives," International Journal of Non-Linear Mechanics, vol. 47, pp. 975-983, 2012.

[10] Y. J. Shen, S. P. Yang, and H. J. Xing, "Dynamical analysis of linear single degree-of-freedom oscillator with fractional-order derivative," Acta Physica Sinica, vol. 61, no. 11, Article ID 110505, 2012. 
[11] Y. Shen, S. Yang, H. Xing, and G. Gao, "Primary resonance of Duffing oscillator with fractional-order derivative," Communications in Nonlinear Science and Numerical Simulation, vol. 17, no. 7, pp. 3092-3100, 2012.

[12] R. Gorenflo and E. A. Abdel-Rehim, "Convergence of the Grünwald-Letnikov scheme for time-fractional diffusion," Journal of Computational and Applied Mathematics, vol. 205, no. 2, pp. 871-881, 2007.

[13] G. Jumarie, "Modified Riemann-Liouville derivative and fractional Taylor series of nondifferentiable functions further results," Computers \& Mathematics with Applications, vol. 51, no. 9-10, pp. 1367-1376, 2006.

[14] M. Ishteva, L. Boyadjiev, and R. Scherer, "On the Caputo operator of fractional calculus and C-Laguerre functions," Mathematical Sciences Research Journal, vol. 9, no. 6, pp. 161170, 2005.

[15] A. B. Malinowska and D. F. M. Torres, "Fractional calculus of variations for a combined Caputo derivative," Fractional Calculus and Applied Analysis, vol. 14, no. 4, pp. 523-537, 2011.

[16] Y. Xu, Y. Li, D. Liu, W. Jia, and H. Huang, "Responses of Duffing oscillator with fractional damping and random phase," Nonlinear Dynamics, vol. 74, no. 3, pp. 745-753, 2013.

[17] J. X. Cao, H. F. Ding, and C. P. Li, "Implicit difference schemes for fractional diffusion equations," Communication on Applied Mathematics and Computation, vol. 27, no. 1, pp. 61-74, 2013.

[18] F. H. Zeng and C. P. Li, "High-order finite difference methods for time-fractional subdiffusion equation," Journal of Computational Physics, vol. 30, no. 4, pp. 491-500, 2013.

[19] A. Chen and C. P. Li, "Numerical algorithm for fractional calculus based on Chebyshev polynomial approximation," Journal of Shanghai University, vol. 18, no. 1, pp. 48-53, 2012.

[20] Z. H. Wang and M. L. Du, "Asymptotical behavior of the solution of a SDOF linear fractionally damped vibration system," Shock and Vibration, vol. 18, no. 1-2, pp. 257-268, 2011.

[21] Z. H. Wang and H. Y. Hu, "Stability of a linear oscillator with damping force of the fractional-order derivative," Science China, vol. 53, no. 2, pp. 345-352, 2010.

[22] T. M. Atanackovic and B. Stankovic, "On a numerical scheme for solving differential equations of fractional order," Mechanics Research Communications, vol. 35, no. 7, pp. 429-438, 2008.

[23] J.-H. Chen and W.-C. Chen, "Chaotic dynamics of the fractionally damped van der Pol equation," Chaos, Solitons and Fractals, vol. 35, no. 1, pp. 188-198, 2008.

[24] J. G. Lu, "Chaotic dynamics of the fractional-order Lü system and its synchronization," Physics Letters A, vol. 354, no. 4, pp. 305-311, 2006.

[25] J. Cao, C. Ma, H. Xie, and Z. Jiang, "Nonlinear dynamics of Duffing system with fractional order damping," Journal of Computational and Nonlinear Dynamics, vol. 5, no. 4, Article ID 041012, 2010.

[26] Z. L. Huang and X. L. Jin, "Response and stability of a SDOF strongly nonlinear stochastic system with light damping modeled by a fractional derivative," Journal of Sound and Vibration, vol. 319, no. 3-5, pp. 1121-1135, 2009.

[27] L. C. Chen and W. Q. Zhu, "The first passage failure of SDOF strongly nonlinear stochastic system with fractional derivative damping," Journal of Vibration and Control, vol. 15, no. 8, pp. 1247-1266, 2009.

[28] C. Luo and X. Wang, "Chaos in the fractional-order complex Lorenz system and its synchronization," Nonlinear Dynamics, vol. 71, no. 1-2, pp. 241-257, 2013.
[29] X. Wang, X. Zhang, and C. Ma, "Modified projective synchronization of fractional-order chaotic systems via active sliding mode control," Nonlinear Dynamics, vol. 69, no. 1-2, pp. 511-517, 2012.

[30] C. Luo and X. Wang, "Chaos generated from the fractionalorder complex Chen system and its application to digital secure communication," International Journal of Modern Physics C, vol. 24, no. 4, Article ID 1350025, 2013.

[31] X.-Y. Wang and J.-M. Song, "Synchronization of the fractional order hyperchaos Lorenz systems with activation feedback control," Communications in Nonlinear Science and Numerical Simulation, vol. 14, no. 8, pp. 3351-3357, 2009.

[32] X.-Y. Wang, Y.-J. He, and M.-J. Wang, "Chaos control of a fractional order modified coupled dynamos system," Nonlinear Analysis: Theory, Methods \& Applications, vol. 71, no. 12, pp. 6126-6134, 2009.

[33] X. Wang and Y. He, "Projective synchronization of fractional order chaotic system based on linear separation," Physics Letters A, vol. 372, no. 4, pp. 435-441, 2008.

[34] X.-Y. Wang and M.-J. Wang, "Dynamic analysis of the fractional-order Liu system and its synchronization," Chaos, vol. 17, no. 3, Article ID 033106, 2007.

[35] H. Q. Wang, Nonlinear Vibration, Higher Education Press, Beijing, China, 1992.

[36] A. Beléndez, D. Méndez, T. Beléndez, A. Hernández, and M. L. Álvarez, "Harmonic balance approaches to the nonlinear oscillators in which the restoring force is inversely proportional to the dependent variable," Journal of Sound and Vibration, vol. 314, pp. 775-782, 2008.

[37] C. M. Bender and L. M. A. Bettencourt, "Multiple-scale analysis of the quantum anharmonic oscillator," Physical Review Letters, vol. 77, no. 20, pp. 4114-4117, 1996.

[38] S. L. Das and A. Chatterjee, "Second order multiple scales for oscillators with large delay," Nonlinear Dynamics, vol. 39, no. 4, pp. 375-394, 2005.

[39] B. T. Floyd, A. M. Ludes, C. Moua, A. A. Ostle, and O. B. Varkony, "Anharmonic oscillator potentials: exact and perturbation results," Journal of Undergraduate Research in Physics, vol. 25, Article ID MS134, 2012.

[40] R. V. Roy, "Averaging method for strongly non-linear oscillators with periodic excitations," International Journal of Non-Linear Mechanics, vol. 29, no. 5, pp. 737-753, 1994.

[41] Z. Rakaric and I. Kovacic, "An elliptic averaging method for harmonically excited oscillators with a purely non-linear non-negative real-power restoring force," Communications in Nonlinear Science and Numerical Simulation, vol. 18, no. 7, pp. 1888-1901, 2013.

[42] J. Cai, "A generalized KBM method for strongly nonlinear oscillators with slowly varying parameters," Mathematical \& Computational Applications, vol. 12, no. 1, pp. 21-30, 2007. 


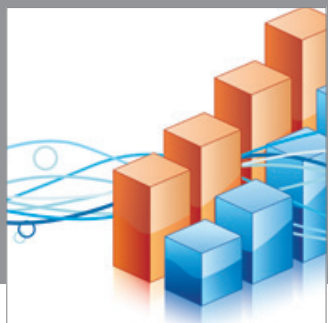

Advances in

Operations Research

mansans

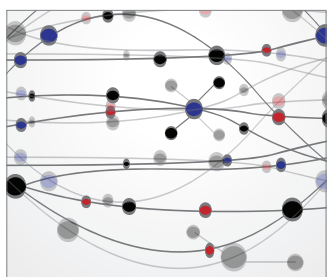

The Scientific World Journal
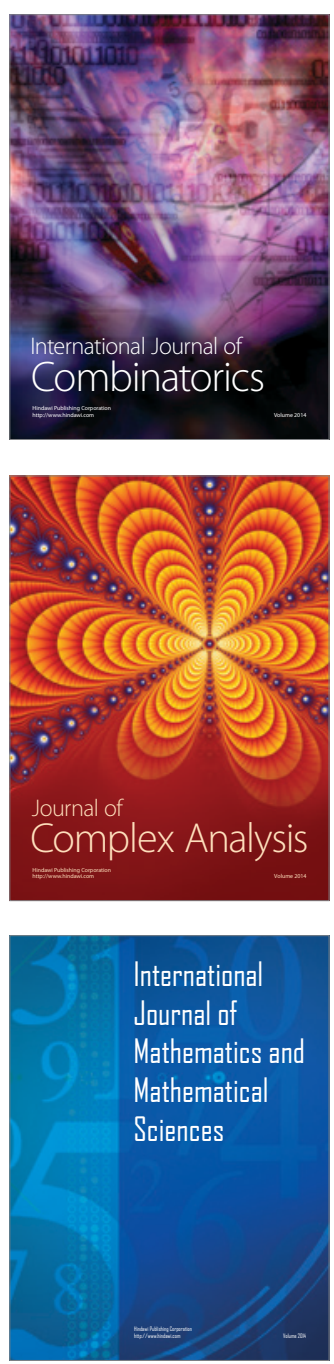
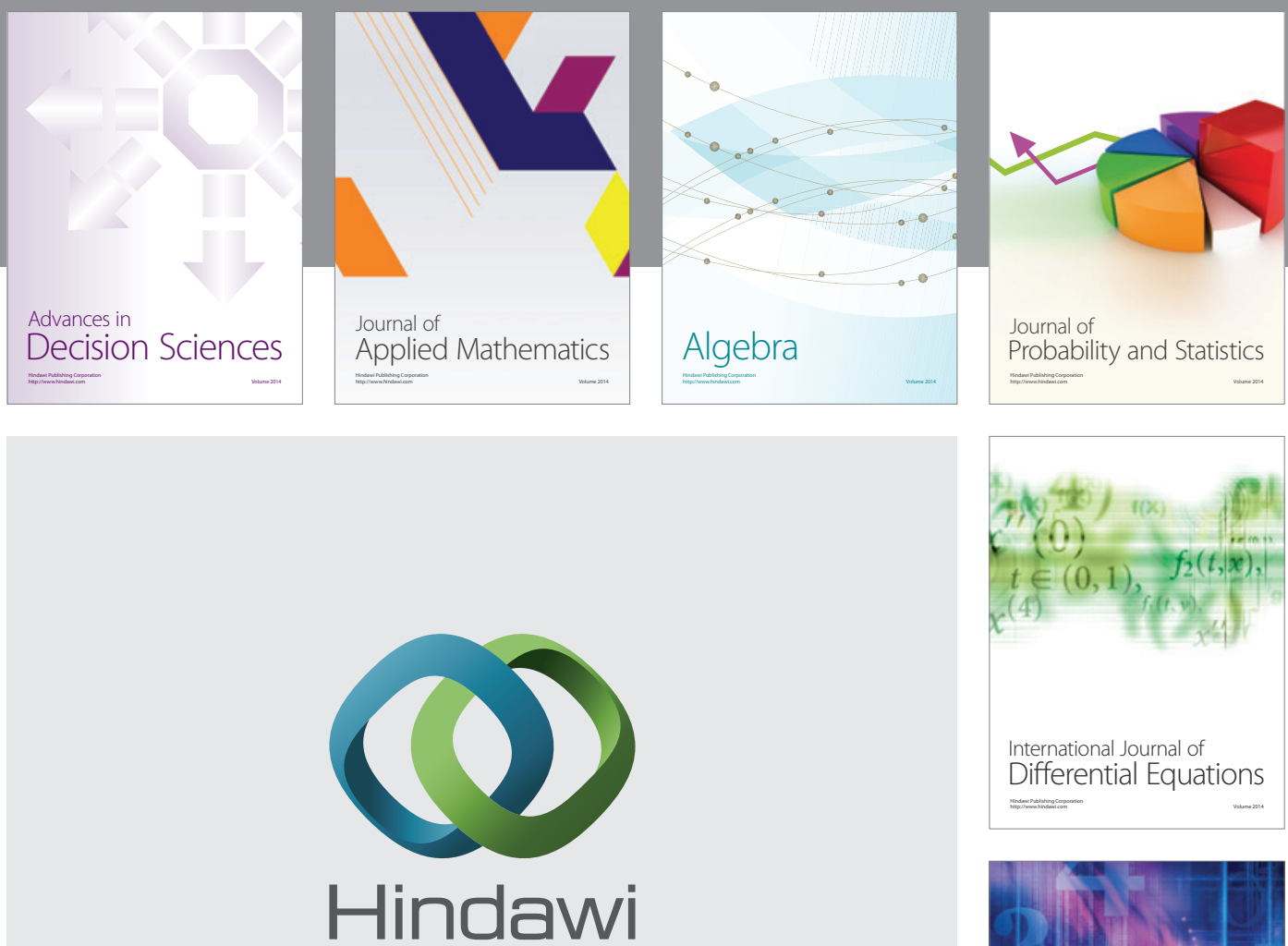

Submit your manuscripts at http://www.hindawi.com
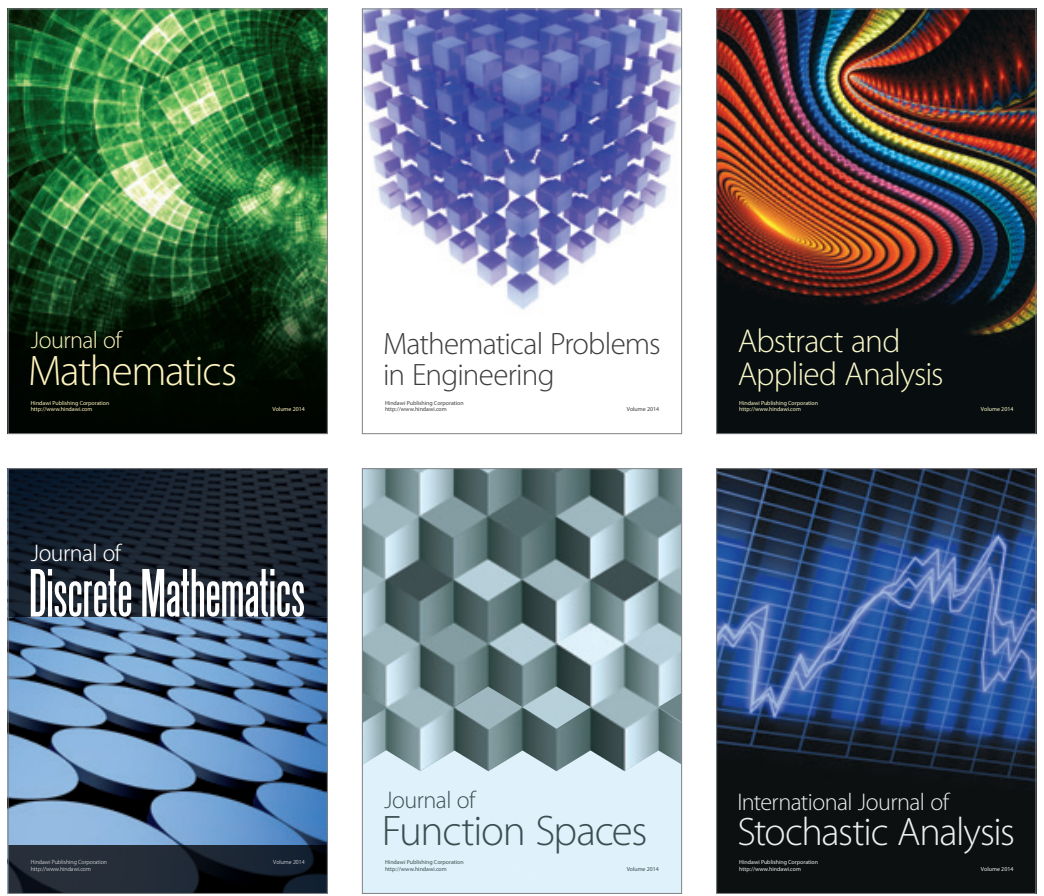

Journal of

Function Spaces

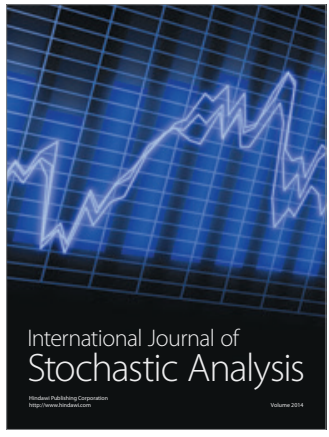

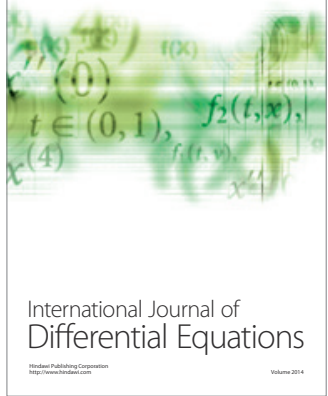
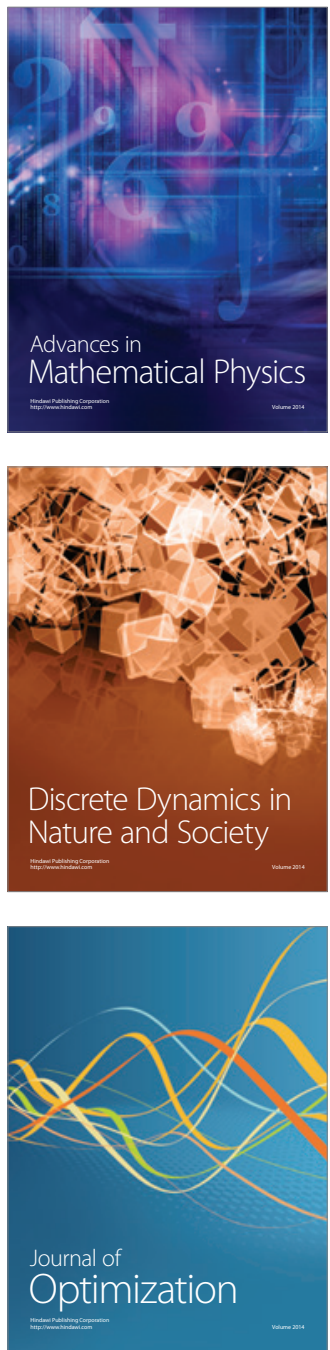